\title{
Thermal conditions during deformation of partially molten crust from TitaniQ geothermometry: rheological implications for the anatectic domain of the Araçuaí belt, eastern Brazil
}

\author{
G. C. G. Cavalcante ${ }^{1,2}$, A. Vauchez ${ }^{2}$, C. Merlet ${ }^{2}$, M. Egydio-Silva ${ }^{1}$, M. H. Bezerra de Holanda ${ }^{1}$, and B. Boyer ${ }^{2}$ \\ ${ }^{1}$ Instituto de Geociências, Universidade de São Paulo, Rua do Lago, 562, 05508-080, São Paulo, Brazil \\ ${ }^{2}$ Géosciences Montpellier, Université de Montpellier 2 \& CNRS Place E. Bataillon, 34095 Montpellier Cedex 05, France \\ Correspondence to: G. C. G. Cavalcante (geanecarol@gmail.com)
}

Received: 2 May 2014 - Published in Solid Earth Discuss.: 28 May 2014

Revised: 29 September 2014 - Accepted: 30 September 2014 - Published: 3 December 2014

\begin{abstract}
During the Neoproterozoic orogeny, the middle crust of the Araçuaí belt underwent widespread partial melting. At the regional scale, this anatectic domain is characterized by a progressive rotation of the flow direction from south to north, suggesting a 3-D deformation of the anatectic middle crust. To better determine whether melt volumes present in the anatectic middle crust of the Araçuaí orogen were large enough to allow a combination of gravity-driven and convergence-driven deformation, we used the titaniumin-quartz (TitaniQ) geothermometer to estimate the crystallization temperatures of quartz grains in the anatectic rocks. When possible, we compared these estimates with thermobarometric estimates from traditional exchange geothermobarometers applied to neighboring migmatitic kinzigites. TitaniQ temperatures range from 750 to $900^{\circ} \mathrm{C}$, suggesting that quartz starts crystallizing at minimum temperatures of $\geq 800^{\circ} \mathrm{C}$. These results, combined with the bulk-rock chemical composition of diatexites, allows the estimation of a minimum of $\sim 30 \%$ melt and a corresponding viscosity of $\sim 10^{9}-10^{10} \mathrm{~Pa}$. Such a minimum melt content and low viscosity are in agreement with interconnected melt networks observed in the field. Considering that these characteristics are homogeneous over a wide area, this supports the finding that the strength of the middle crust was severely weakened by extensive partial melting, making it prone to gravitydriven flow and lateral extrusion.
\end{abstract}

\section{Introduction}

Geophysical profiles and geological observations in hot orogens suggest that large volumes of the middle to lower crust were partially molten during deformation (e.g., Chen et al., 1996; McNamara et al., 1996; Nelson et al., 1996; Alsdorf et al., 1998; Unsworth et al., 2005). Indeed, the presence of even a small fraction of melt may dramatically reduce the strength of rocks and affect their tectonic behavior (e.g., Rosenberg and Handy, 2005; Labrousse et al., 2011). When partial melting is widespread, it may result in the development of a thick, low-strength layer in the crust that deeply alters its rheological behavior during orogeny. In this case, numerical models (e.g., Beaumont et al., 2004) suggest that, provided the viscosity of the lower to middle crust is low enough (e.g., $\leq 10^{19}$ Pas Beaumont et al., 2004; $\sim 10^{17}$ Pas, Grujic et al., 1996), complex deformation patterns may occur in response to a combination of tectonic and gravitational forces.

The Araçuaí belt of eastern Brazil is one of several Neoproterozoic belts that involve a large anatectic domain (Oliveira et al., 2000; Vauchez et al., 2007; Petitgirard et al., 2009; Cavalcante et al., 2013). Most previous studies of the Araçuaí belt, including those studying the anatectic domain, placed emphasis on the characterization of strain distribution and/or magmatic episodes (e.g., Pedrosa-Soares et al., 2006; Vauchez et al., 2007; Mondou et al., 2012; Cavalcante et al., 2013); only few data on the thermal conditions and partial melting during deformation are available. Preliminary pressure $(P)$ and temperature $(T)$ estimates in mylonites from the western part of the belt (e.g., Petitgirard et 
al., 2009) suggest high-temperature $\left(\sim 750^{\circ} \mathrm{C}\right)$, low-pressure $(\sim 600 \mathrm{MPa})$ synkinematic conditions.

Recently, we have shown that the anatectic domain in the eastern Araçuaí belt displays a complex 3-D strain distribution that we tentatively interpreted as resulting from the deformation of a low-stiffness anatectic middle crust in response to a combination of tectonic forces from the South America-Africa collision and gravity-driven flow (Cavalcante et al., 2013). However, temperature estimates from the anatectic domain are lacking, and such an interpretation assumes that rheological critical conditions that allowed the development of gravity-driven flow in the anatectic middle to lower crust were reached during orogeny. It is, however, difficult to evaluate, even roughly, the synkinematic viscosity of most anatectic domains without estimating equilibrium temperatures and evaluating the melt proportion present in the anatectic rocks during deformation.

Estimates of temperatures were performed using the titanium-in-quartz (TitaniQ) geothermometer developed by Wark and Watson (2006), on migmatitic rocks of the anatectic domain. We have chosen this geothermometer because it requires analysis of only one phase, quartz, its high potential to record temperatures in a major rock-forming mineral (e.g., Negrini et al., 2014), and because anatexites often do not display adequate mineralogical assemblage required for exchange geothermometers. Furthermore, microstructural observations and microprobe analysis of minerals in anatexites, migmatitic granulite and kinzigites indicate that quartz crystallized in equilibrium with rutile, and therefore represents a natural case similar to laboratory experiments performed for the calibration of the TitaniQ geothermometer. To test the reliability of the approach, migmatitic kinzigites with suitable mineralogical assemblages that bound the anatexites eastward were analyzed with both conventional thermobarometry and TitaniQ thermometry. In addition, as the titanium concentration in quartz is pressure sensitive, we have also estimated the temperatures using the calibrations by Thomas et al. (2010) and Huang and Audétat (2012). Finally, to assess the probability of a contribution of gravity-driven flow to the overall deformation, the temperature estimates and bulk rock composition of anatectic magma were used to evaluate the degree of partial melting of the anatectic middle crust during deformation. This evaluation, combined with field and microstructural observations, allowed us to constrain the viscosity of the anatectic crust more reliably and to test whether it was lower than the viscosity threshold below which topographic loading starts contributing to tectonic forces.

\section{Geological setting}

The convergence between the South American and African continents amalgamated lithospheric blocks of different compositions and ages and formed the Ribeira-AraçuaíWest Congo orogen (e.g., Trompette, 1994; Monié et al., 2012, Fig. 1). The Araçuaí belt and its southward con-

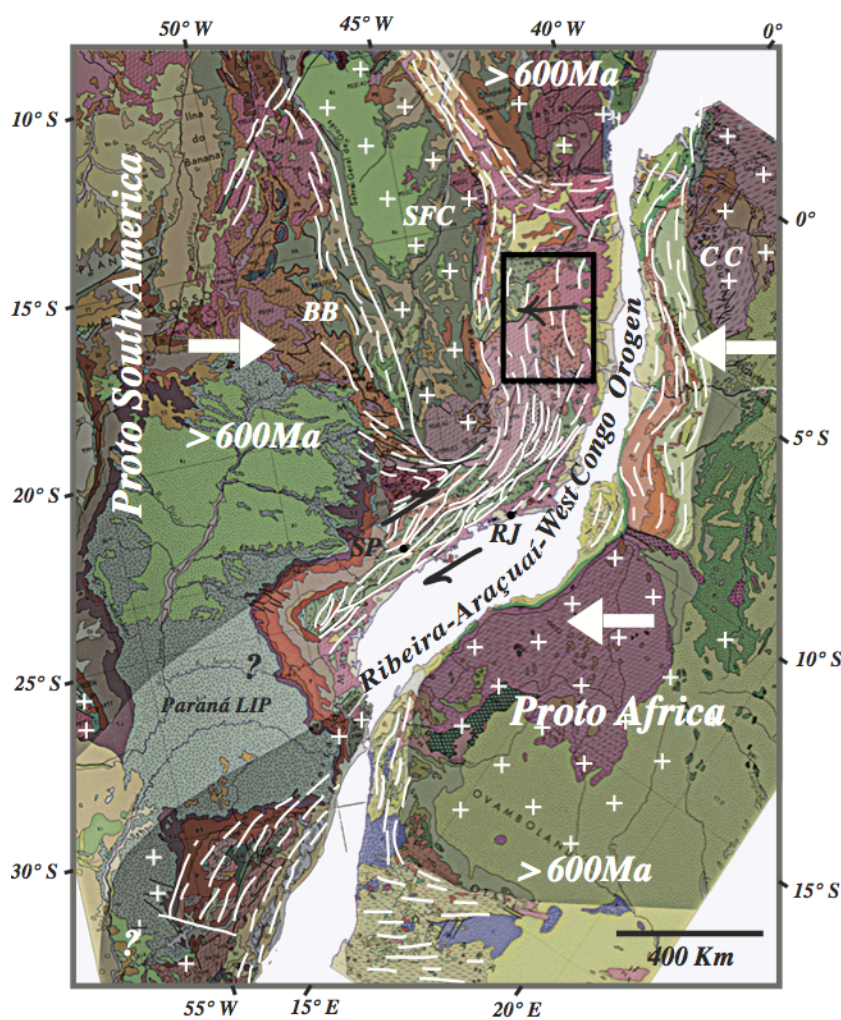

Figure 1. Schematic reconstitution of the position of South America and Africa before the opening of the South Atlantic (Vauchez et al., 2007). Shaded areas represent domains where collision occurred before $\sim 600 \mathrm{Ma}$ (white crosses: cratonic domains; white lines: Neoproterozoic belts). The area delimited by the rectangle represents the location of Fig. 2. Arrows show the convergence between South America and Africa. Small black arrows show the main kinematics. CC: Congo craton; BB: Brasilia belt; SP: São Paulo; RJ: Rio de Janeiro.

tinuation, the Ribeira belt, were formed by Neoproterozoic convergence between the São Francisco and Congo cratons. The Araçuaí belt is characterized by the dominant thrusting of high-temperature metamorphic allochthonous units on the São Francisco craton and subordinate orogen-parallel transcurrent motions, whereas the Ribeira belt is characterized by dominant orogen-parallel dextral transcurrent shearing combined with thrusting normal to the belt (Trompette, 1994; Egydio-Silva et al., 2002; Schmitt et al., 2004).

Schematically, the Araçuaí belt is divided into three lithological domains (Oliveira et al., 2000; Vauchez et al., 2007): the western mylonitic unit, the central plutonic unit and the eastern anatectic unit (Fig. 2a). The mylonitic domain is composed of high-temperature metasedimantary mylonites injected by a large volume of leucogranite sills. These rocks were thrust westward (top to west) onto the paraautochthonous metasediments of the São Francisco craton (Cunningham et al., 1996; Petitgirard et al., 2009). Eastward, the central plutonic domain comprises syncollisional 


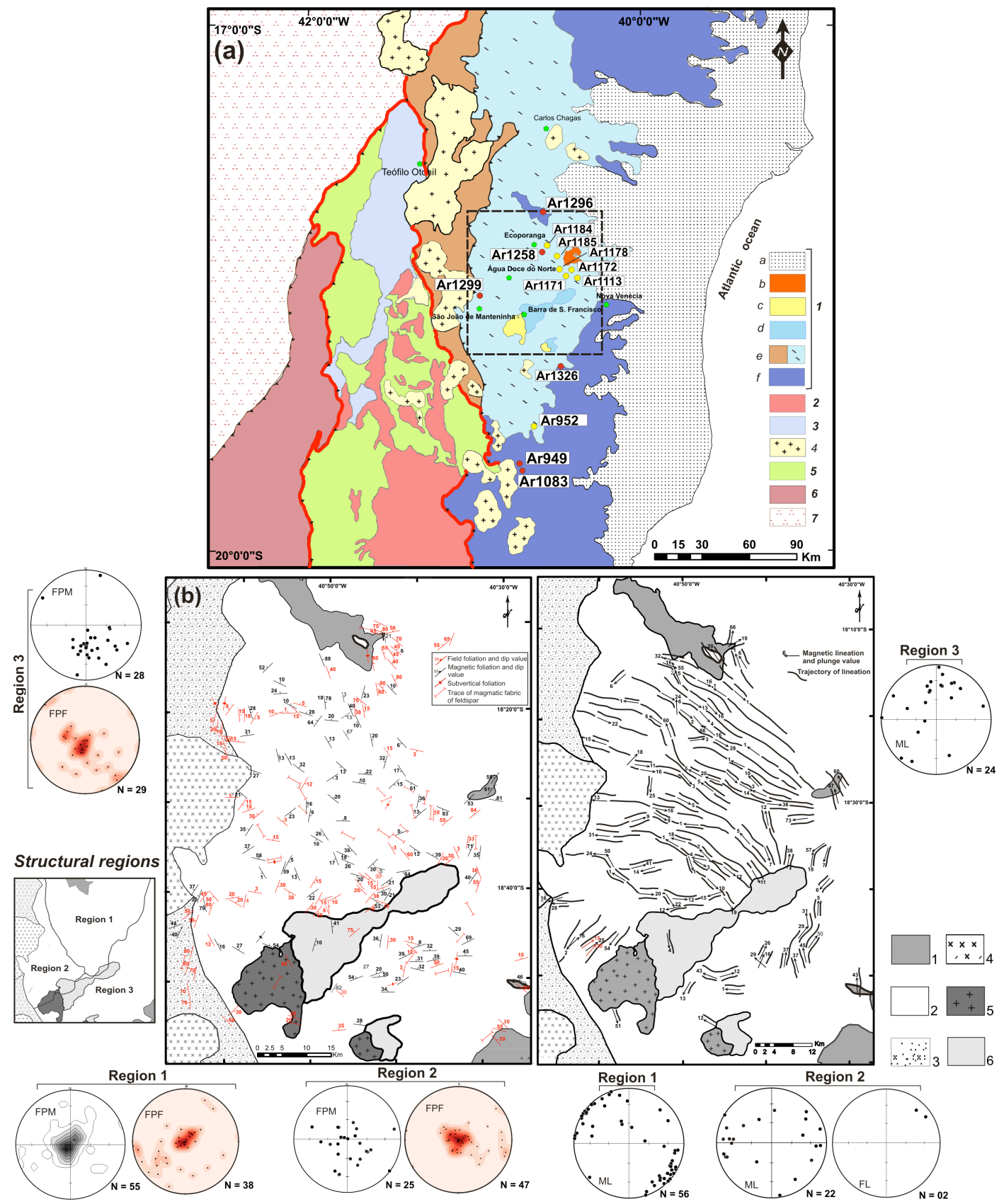

Figure 2. (a) Simplified geological map (after Oliveira et al., 2000 , and Vauchez et al., 2007) showing the main domains of the Araçuaí belt and the location of the samples selected for this study (red = samples analyzed by TitaniQ and yellow = samples of geochemistry analysis): the eastern domain (1) comprises a thick $(>10 \mathrm{~km}$ ) layer of diatexites, metatexites and anatectic granites (e) associated with migmatitic granulites (f) and intruded on by granite (c) and charnockite (d). (a) and (b) represent the Phanerozoic cover. The central domain comprises a sycollisional magmatic complex ( 2 = Galiléia batholith; 3 = São Vitor tonalite) intruding in HT metasediments (5). The western domain involves HT metasedimentary mylonites injected by abundant synkinematic leucocratic magma (6) thrust upon the para-autochthonous metasedimentary cover of the São Francisco craton (7). Late orogenic ( $\sim 520 \mathrm{Myr}$, Mondou et al., 2012) porphyritic granitoids associated with charnockites (4) intrude on the stack of allochthonous units. The red line represents the boundary between lithological domains. The green pentagons represent cities. The dashed box shows the location of (b) (structural map showing the AMS and the field measurements across the anatectic domain from Cavalcante et al., 2013): left foliations; the small map on the left shows the three main structural regions as defined by their magnetic lineation pattern. The stereoplots FPM and FPF for each structural region represent the foliation poles obtained from the AMS (k3) and measured in the field, respectively. Right: lineation and lineation traces illustrating the magmatic flow. The ML (k1) and FL stereoplots for each structural region represent the lineation obtained from the AMS and measured in the field, respectively. All the stereoplots are represented in the lower hemisphere of the equal-area projection. 1, 2, 3, 4, 5 and 6 are, respectively, 1f, 1e (Carlos Chagas anatexite), 1e, 4, 1c and 1d of (a). 
tonalite and granodiorite bodies deformed in the magmatic state (Mondou et al., 2012). The eastern anatectic domain is dominated by a variety of anatexites and anatectic granites that result from pervasive partial melting of metasedimentary rocks under mid-crustal $P$ and $T$ conditions (Vauchez et al., 2007). This anatectic domain is $\sim 300 \mathrm{~km}$ long and 50 $100 \mathrm{~km}$ wide. Migmatitic granulite forms rafts embedded in the anatexites. Migmatitic kinzigites occur east and southeast of the anatexites; they progressively grade westward to diatexites and aluminous granites, suggesting a transition from kinzigites to anatexites. Structural mapping in the anatectic unit using the anisotropy of magnetic susceptibility (AMS) highlighted a complex pattern of lineation, the trend of which rotates from WSW-ENE in the southern part of the study area to NW-SE in the northern part, and to N-S in the easternmost part (Cavalcante et al., 2013; Fig. 2b). Flow in varying directions is expected during deformation of a weakened crust that cannot support the topographic load; such deformation "is analogous to pressing on an egg sandwich: the soft filling is squeezed out and emerges at the edge of the sandwich and through any holes in the bread" (Jamieson et al., 2011). Considering the progressive transition between the various dominant orientations of lineation together with evidence supporting the finding that, independently of the flow direction, the fabric was acquired in the magmatic state (Cavalcante et al., 2013), led us to suggest that the complex deformation of the anatexites results from a single, probably protracted, tectonic event.

Thermochronological data from the mylonitic domain suggest that this region remained hot $\left(750\right.$ to $\left.>500^{\circ} \mathrm{C}\right)$ for tens of millions of years (580-530 Ma) due to a low cooling rate $\left(\leq 5^{\circ} \mathrm{CMyr}^{-1}\right.$; Petitgirard et al., 2009). Conventional thermobarometric estimates performed by Munhá et al. (2005) in migmatitic gneisses from the southern part of the Araçuaí belt suggest that the metamorphic peak and partial melting took place at temperatures of $820 \pm 30^{\circ} \mathrm{C}$ and at pressures of $650 \pm 50 \mathrm{MPa}$.

\section{Samples: location and description}

Thirteen samples were selected from the anatectic unit (Fig. 2a). Six samples were used for TitaniQ analyses (Ar1258, Ar1299, Ar1326, Ar1296, Ar949 and Ar1083), and seven samples for geochemistry analyses (Ar1113, Ar952, Ar1171, Ar1172, Ar1178, Ar1184 and Ar1185). All these samples are anatexites, however, with different volumes of melt and composition. Nine samples come from the diatexites (Ar1258, Ar1299, Ar1113, Ar952, Ar1171, Ar1172, Ar1178, Ar1184 and Ar1185), one from the metatexite (Ar1326), one from the migmatitic granulite of the northern domain (Ar1296), and two from the migmatitic kinzigites of the southeastern domain (Ar949 and Ar1083). At the outcrop scale, the diatexites display a clear magmatic foliation marked by the preferred alignment of biotite and feldspar
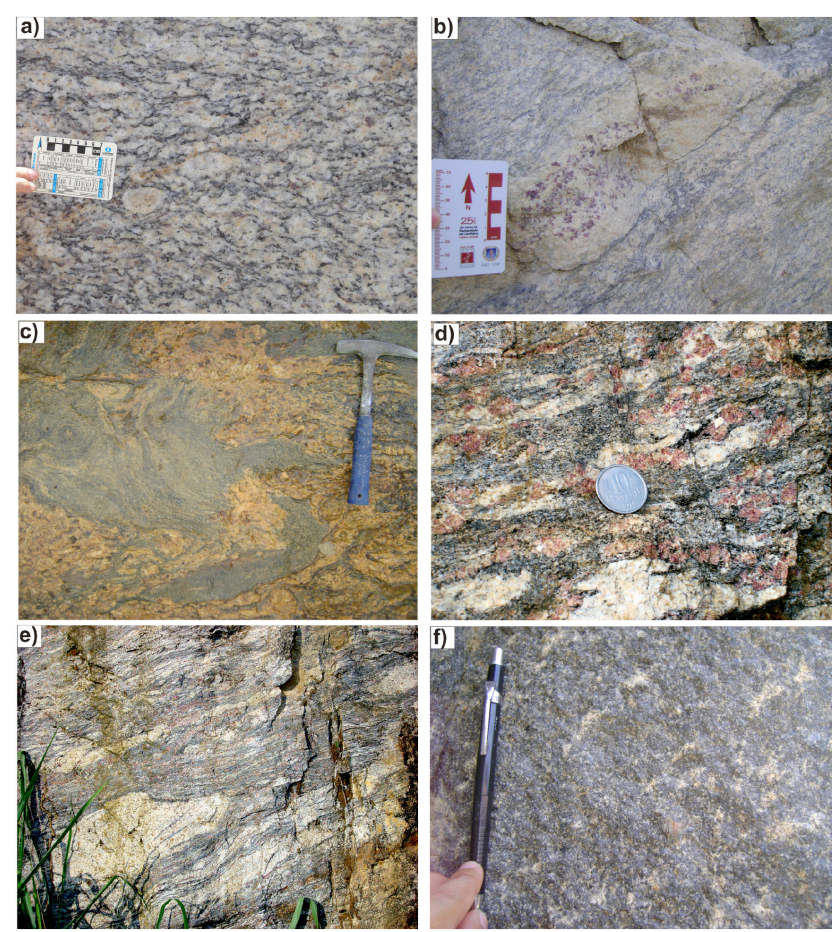

Figure 3. Pictures of outcrops representative of the studied rocks: (a) an example of diatexite with well-marked magmatic foliation characterized by the preferred alignment of biotite and feldspar; (b) diatexite with leucosome rich in garnet and feldspar; (c) diatexite displaying a scholle feature. (d) and (e): migmatitic kinzigites displaying stromatic leucosomes embedded between gneissic foliations; (f) a migmatitic granulite with small pockets of melt.

grains (Fig. 3a). In situ leucosomes are frequently observed in metatexites that preserve a gneissic banding (Fig. 4). Migmatitic features such as stromatic, nebulitic and schlieren leucosomes, rich in garnet and feldspar, are also common (Fig. 3b, c).

The migmatitic kinzigites display a gneissic banding characterized by alternating felsic and mafic layers. Stromatic and coarser-grained leucosomes, rich in feldspar, garnet and quartz, are embedded parallel to banding (Fig. 3d, e).

The migmatitic granulite is mafic in composition and does not show a clear macroscopic fabric. It does, however, contain narrow strings of leucosomes pointing to limited partial melting (Fig. 3f).

\section{Petrographic observations}

Diatexites, metatexites and migmatitic kinzigites are composed of K-feldspar, quartz, plagioclase, biotite, garnet, cordierite, sillimanite, ilmenite and rutile. The migmatitic granulite also contains orthopyroxene \pm magnetite. Most minerals do not or rarely show evidence of intracrystalline deformation; this suggests a predominantly magmatic fabric free of superimposed solid-state deformation. 

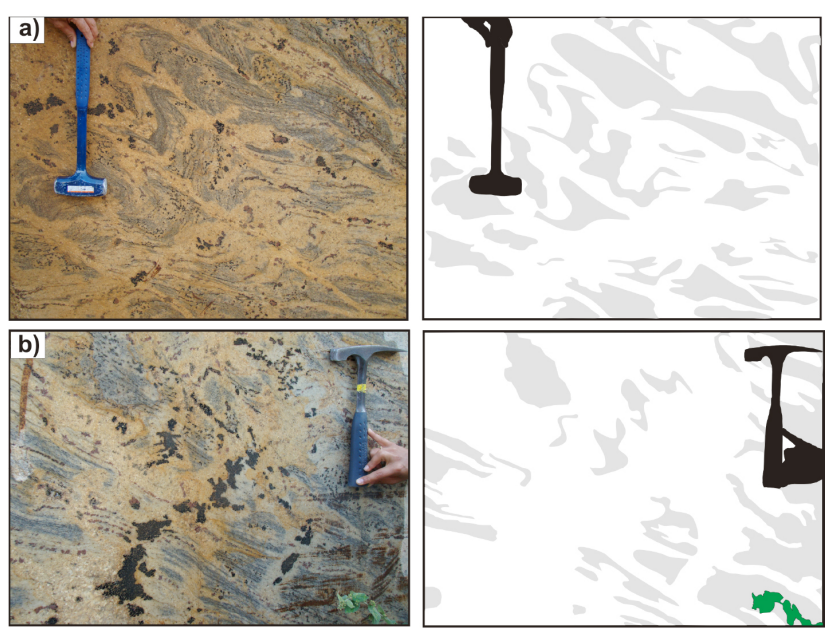

Figure 4. Pictures (left) and schematic drawing (right) of partially molten kinzigites of the Araçuaí belt. White represents the in situ leucosomes and gray represents the residuum and/or paleosome portions.

Quartz usually displays interstitial shapes (Fig. 5a), although this is less frequent in the migmatitic granulite that contains less quartz. It has irregular grain boundaries, with sizes ranging from $\sim 200 \mu \mathrm{m}$ to $\sim 2 \mathrm{~mm}$, and is mostly free of substructure, with the exception of an undulose extinction or faint subgrain boundaries (Fig. 5b).

Plagioclase reaches $\sim 0.4 \mathrm{~cm}$ in size, and predominantly displays subeuhedral shapes; in many cases, it is surrounded by interstitial films of quartz along grain boundaries (Fig. 5c). In the migmatitic granulites, many crystals of plagioclase display deformation twins, attesting to the high temperature deformation during partial melting (Fig. 5d).

$\mathrm{K}$-feldspar is $\sim 0.3 \mathrm{~cm}$ in size, and displays curved, lobate and straight grain boundaries. It often exhibits myrmekites, and is free of evidence of intracrystalline deformation.

Biotite displays a strong preferred orientation (Fig. 5a, e). It is euhedral, subeuhedral and platy in shape, with straight to curved grain boundaries. It tends to arch around large Kfeldspars (Fig. 5f). In the migmatitic kinzigites, a few kinked biotites are observed.

Sillimanite is acicular and garnet is usually rounded, but locally elongate parallel to foliation, with frequent inclusions of quartz, biotite, ilmenite and rutile.

\section{TitaniQ temperature estimates}

The TitaniQ geothermometer is based on substitution of $\mathrm{Si}$ by $\mathrm{Ti}$ in quartz. Since the substitution is dependent on temperature (Wark and Watson, 2006), Ti concentration in quartz is correlated with its formation temperature. TitaniQ has already been applied to characterize thermal histories of migmatitic (Storm and Spear, 2009), mylonitic (Kohn and Northrup, 2009; Grujic et al., 2011) and volcanic rocks (e.g.,
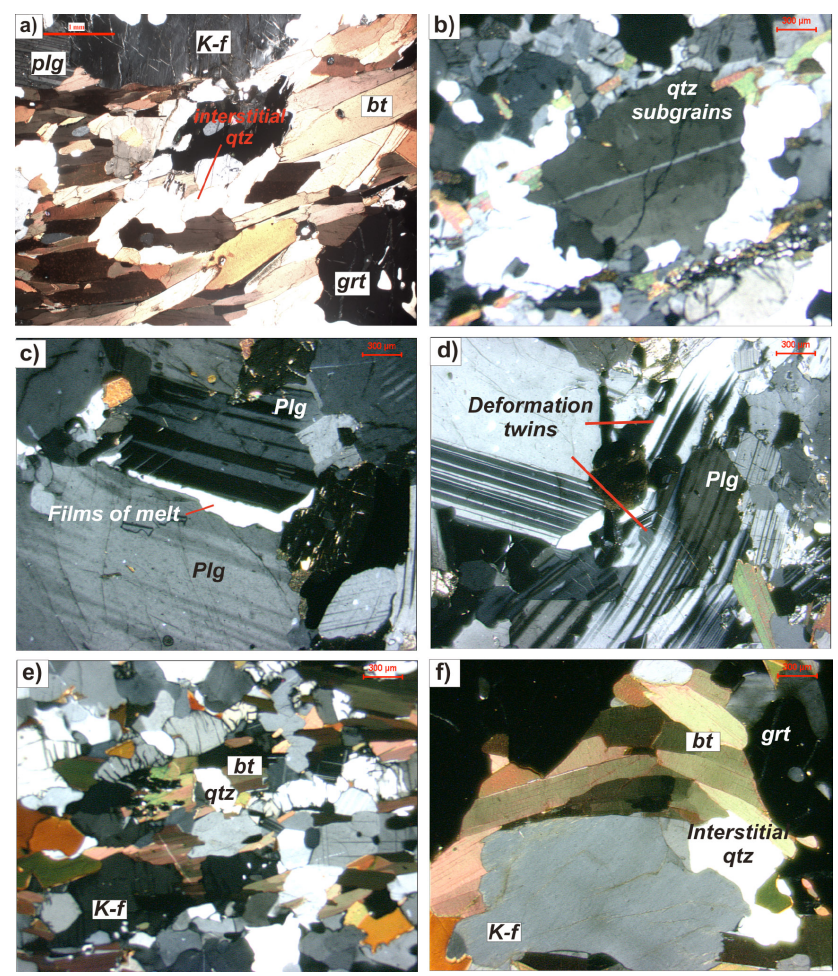

Figure 5. Photomicrographs of anatexites and migmatitic granulites. (a) Large interstitial quartz and preferred orientation of biotite characterizing the magmatic foliation, (b) quartz subgrains representing the record of the intracrystalline deformation, (c) film of quartz crystallized along grain boundaries that likely represent former films of melt, (d) deformation twins in plagioclase feldspar, (e) preferred orientation of biotite grains, and (f) large K-feldspar grains associated with interstitial quartz and arched biotite.

Ehrlich et al., 2012). To develop this geothermometer, Wark and Watson (2006) synthesized quartz in the presence of rutile and aqueous fluid or hydrous silicate melt at temperatures ranging from 600 to $1000{ }^{\circ} \mathrm{C}$ and $1.0 \mathrm{GPa}$ of pressure. This geothermometer relates the temperature $(T)$ of crystallization to the Ti content of quartz according to

$\log \left(\operatorname{Ti}_{\text {quartz }}\right)=5.69-3765 / T$

where $\mathrm{Ti}_{\text {quartz }}$ is the $\mathrm{Ti}$ concentration in quartz in ppm and $T$ is the temperature in K. No adjustment was necessary for this equation because both rutile and ilmenite were present in the analyzed samples. Wark and Watson (2006) calibration however does not take into account the pressure dependence of Ti concentrations in quartz. Thomas et al. (2010), then Huang and Audétat (2012), experimentally calibrated a TitaniQ thermobarometer. Thomas et al. (2010) data are fitted 
by the equation

$$
\begin{aligned}
R T \ln \mathrm{X}_{\mathrm{TiO}_{2}}^{\mathrm{Quartz}} & =-60952+1520 \cdot T(\mathrm{~K})-1741 \cdot P(\mathrm{kbar}) \\
& +R T \ln a_{\mathrm{TiO}_{2}},
\end{aligned}
$$

where $R$ is the gas constant $8.3145 \mathrm{~J} \mathrm{~K}^{-1}, T$ is the temperature in Kelvin, $\mathrm{X}_{\mathrm{TiO}_{2}}^{\mathrm{Quartz}}$ is the mole fraction of $\mathrm{TiO}_{2}$ in quartz, and $a_{\mathrm{TiO}_{2}}$ is the activity of $\mathrm{TiO}_{2}$ in the system. Those from the Huang and Audétat (2012) experiments are fitted by

$$
\begin{aligned}
\log \operatorname{Ti}(\mathrm{ppm}) & =-0.27943 \times 10^{4} / T \\
& -660.53\left(P^{0.35} / T\right)+5.6459,
\end{aligned}
$$

where $T$ is given in $\mathrm{K}$ and $P$ in kbar.

We have therefore also calculated the crystallization temperatures using these two calibrations for comparison.

\section{Material and procedure}

Microprobe analysis and backscattered electron images were performed and produced with a CAMECA SX-100 electron probe instrument equipped with five wavelength-dispersive $\mathrm{X}$-ray spectrometers. Some of the X-ray spectrometers are equipped with a large, high-efficiency Johansson crystal. Before microprobe analysis, the selected samples were imaged by cathodoluminescence, and no evidence of zoning was observed (Fig. 6). All quartz grains display an intense homogeneous dark blue color, suggesting that multiple thermal events are unlikely. We have selected large and/or interstitial quartz grains $(\geq 0.3 \mathrm{~mm})$ that have similar aspects, no inclusion and no evidence of intracrystalline deformation or microfracture.

Four to five quartz grains from each sample were analyzed. Microprobe analyses were made along transverse profiles with a spacing of at least $120 \mu \mathrm{m}$ (Fig. 7), and preferentially in areas free of rutile and impurities. More than 370 Ti-in-quartz analyses were performed on samples from the anatectic domain.

\subsection{Analytical procedures}

Accurate characterization of trace elements using electron probe micro-analysis (EPMA) requires optimal analytical conditions (e.g., Merlet and Bodinier, 1990; Bodinier et al., 1996; Kalfoun et al., 2002a, b). The choice of the accelerating voltage value is essential, because it influences the $\mathrm{X}$-ray emission yield and bias. The best spatial resolution is obtained with a low accelerating voltage, whereas higher voltages are needed for transition and trace elements. These constraints require a compromise between X-ray line, peak counting statistics, background subtraction, electron beam size, beam drift, quantification uncertainty, stray radiation and sample damage. In order to find the optimum balance, recent developments in microanalysis, such as spectrum simulation and X-ray emission modeling (Fournier et al., 1999; Kalfoun et al., 2002a, b), were taken into account.
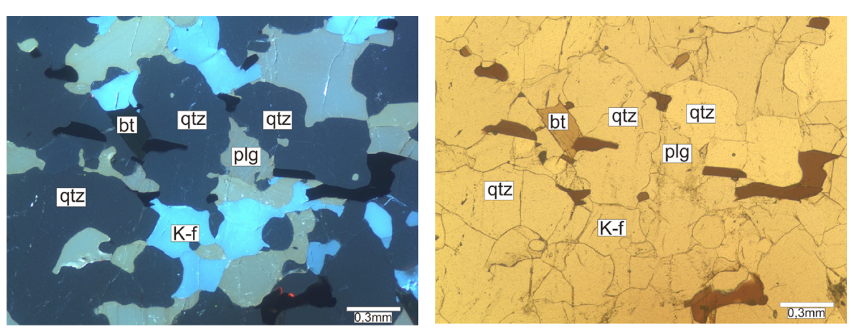

Figure 6. CL (right) and true-color (left) images of the migmatitic kinzigite (sample Ar949) displaying the intense homogenous dark blue of quartz grains.
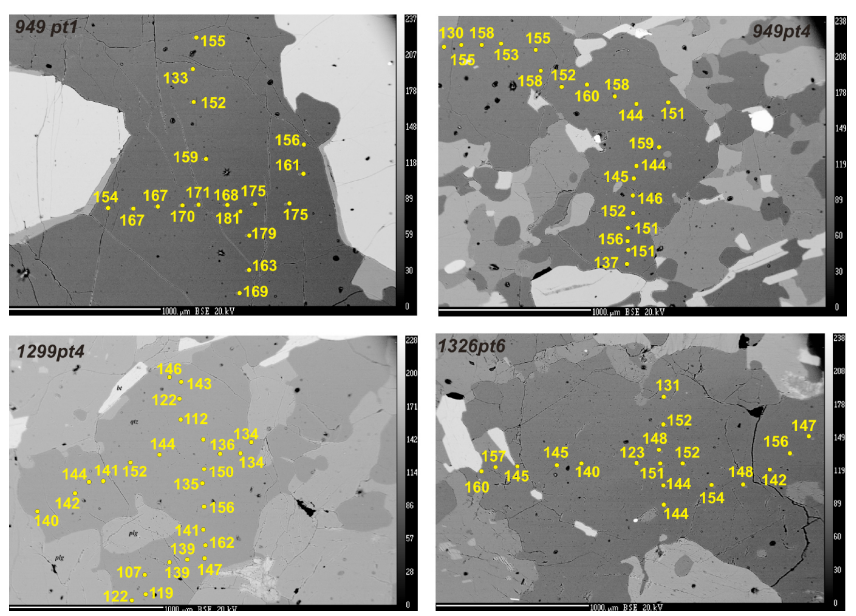

Figure 7. Spots along transverse profiles and respective titanium concentrations (ppm) in quartz from migmatitic kinzigite (samples Ar949 and Ar1326) and from diatexite (Ar1299).

Traces of titanium in quartz are relatively easy to identify, but care must be taken against sample damage and Bremsstrahlung fluorescence (braking radiation) of Ti atoms in close proximity to rutile (Wark and Watson, 2006), ilmenite and biotite. The Bremsstrahlung effect is a form of secondary fluorescence produced by elastic interaction of the $15 \mathrm{kV}$ incident electrons with the quartz lattice (e.g., Bastin et al., 1984) that can be calculated by Monte Carlo simulation. At $20 \mathrm{kV}$ accelerating voltage, a minimum gap of 200 microns between these minerals is required in order to avoid erroneous estimates of Ti concentrations.

Concentrations were measured using an accelerating voltage of $20 \mathrm{kV}$, a beam current of $200 \mathrm{nA}$ and a 50 -micron defocused probe to reduce irradiation damage. An electron beam current was selected to compromise between a high Xray counting rate and minimal sample damage. The Ti concentration was determined by simultaneously counting Ti Kalpha X-ray lines using three spectrometers with large pentaerythritol (LPET) crystals in a Johansson configuration and averaging the results.

Bremsstrahlung background was subtracted using a method that combines the measured and calculated 
background spectra from samples for which neither spectral lines nor absorption edges were observed in the region of interest (Kalfoun et al., 2002). The peak and background acquisition time for each point for all elements and all five spectrometers was $480 \mathrm{~s}$. The counting time of $480 \mathrm{~s}$ is the maximum time that a defocused beam of 50 microns can shine on a sample without damaging it significantly. The corresponding integration time and beam current provide a typical statistical error (peak counting) of approximately $1.5 \%$ for a $100 \mathrm{ppm}$ Ti concentration, and thus a $7 \mathrm{ppm}$ limit of detection. Concentrations are obtained from raw intensities by using the X-PHI quantification procedure (Merlet, 1994). Natural $\mathrm{SiO}_{2}$ and $\mathrm{TiO}_{2}$ are used as standards for $\mathrm{Si}$ and $\mathrm{Ti}$, respectively.

The global uncertainty of Ti concentrations at a single point, obtained by adding the random and systematic uncertainties in quadrature, is $\pm 6 \%(2 \sigma)$ at $\geq 100 \mathrm{ppm}$; these estimates consider errors from peak counting $(1.5 \%)$, background estimates $(2 \%)$, sample damage and contamination $(1.5 \%)$, and uncertainties in standard composition and quantification procedures $(1 \%)$.

\subsection{Results}

The $\mathrm{Ti}$ content in quartz from diatexites, metatexites, migmatitic kinzigites and granulite, and the calculated temperatures, are presented in Table 1 and Fig. 8. Temperature was calculated from each analytical spot. The average Ti content is $\sim 129 \mathrm{ppm}$ in anatexites (diatexites and metatexite), $\sim 142 \mathrm{ppm}$ in migmatitic kinzigites and $\sim 112 \mathrm{ppm}$ in migmatitic granulite. The average crystallization temperature calculated for all quartz grains from the selected samples ranges from 840 to $900^{\circ} \mathrm{C}$ in Huang and Audétat's (2012) calibration, from 670 to $730^{\circ} \mathrm{C}$ in the Thomas et al. (2010) calibration, and from 700 to $810^{\circ} \mathrm{C}$ in Wark and Watson's (2006) equation. Single quartz crystals display a rather homogenous distribution of titanium, and consequently uniform temperature values (Table 1; see values for individuals grains). Specimen Ar1296, a mafic granulite, constitutes an exception, with temperature averages for grains 1 and 3 (ar1296pt01 and ar1296pt03 in Table 1) exhibiting a variation of greater than $100^{\circ} \mathrm{C}$. This rock is derived from a mafic magmatic protolith in which the presence of a rather small amount of interstitial quartz and a large amount of plagioclase locally showing deformation twins (Fig. 5d) suggests limited partial melting during high-temperature, solid-state deformation.

For the diatexites and metatexites, calculated temperature averages obtained from the Huang and Audétat (2012), Thomas et al. (2010) and Wark and Watson (2006) calibrations are, respectively, $848 \pm 20^{\circ} \mathrm{C}, 676 \pm 10^{\circ} \mathrm{C}$ and $747 \pm$ $15^{\circ} \mathrm{C}$ for sample $\operatorname{Ar} 1258,868 \pm 20^{\circ} \mathrm{C}, 703 \pm 10^{\circ} \mathrm{C}$ and $780 \pm 16^{\circ} \mathrm{C}$ for sample $\operatorname{Ar} 1299$ from the central part of the study area, and $881 \pm 20^{\circ} \mathrm{C}, 715 \pm 10^{\circ} \mathrm{C}$, and $792 \pm 17^{\circ} \mathrm{C}$ for sample Ar1326 from the southeast, and for the partially molten kinzigites, high temperature averages are observed, $884 \pm 20^{\circ} \mathrm{C}, 717 \pm 10^{\circ} \mathrm{C}$ and $794 \pm 17^{\circ} \mathrm{C}$ for sample Ar949 and $877 \pm 20^{\circ} \mathrm{C}, 710 \pm 10^{\circ} \mathrm{C}$ and $787 \pm 17^{\circ} \mathrm{C}$ for sample Ar1083.

\section{Exchange reaction thermobarometry}

Thermobarometric estimates using exchange reactions were determined from the migmatitic kinzigites (samples Ar949, Ar1083 and Ar1076) for comparison with the temperatures determined with the TitaniQ thermometry. Sample Ar1076 collected close to site Ar1083 was not analyzed for TitaniQ thermometry, but was used for exchange thermobarometry due to an adequate mineral assemblage.

Temperatures were obtained using the geothermometer developed by Ferry and Spear (1978) and Bhattacharya et al. (1988, 1991). Pressures were estimated using the garnet-plagioclase-biotite-quartz (GPBQ) geobarometer ( $\mathrm{Wu}$ et al., 2004). We chose these geothermobarometers by considering the textural characteristics of the analyzed mineral pairs, i.e., those displaying equilibrium relations expected on the temperature interval on which the geothermobarometers were calibrated. The resulting $P-T$ estimates are presented in Table 2.

Cores and rims of garnet, biotite, plagioclase, cordierite and orthopyroxene were analyzed (Table 3). Garnet-biotite, garnet-orthopyroxene and garnet-cordierite geothermometers and a GPBQ geobarometer applied to analyses from the cores of the crystals suggest that the minimum temperatures for peak metamorphic conditions recorded in migmatitic kinzigites range from 720 to $840 \pm 50^{\circ} \mathrm{C}$, and pressure from 650 to $700 \pm 100 \mathrm{MPa}$. These temperatures are close to those obtained with TitaniQ for the same samples. They usually are between the temperatures calculated using the Wark and Watson (2006) and Huang and Audétat (2012) calibrations: they are however higher than those computed with the Thomas et al. (2010) calibration. Significantly lower temperatures $\left(550-780^{\circ} \mathrm{C}\right)$ obtained in garnet, biotite and opx rims likely represent cooling during retrogressive metamorphic conditions. However, more data are required to constrain the significance of these values better.

\section{Discussion}

Paleotemperatures recorded by the anatectic rocks of the eastern part of the Araçuaí belt have been calculated from Ti content in quartz. Individual crystals display rather homogeneous compositions (Fig. 7), suggesting that Ti diffusion after crystallization was limited. For instance, analysis from sample AR949 shows a good correlation between temperatures estimated from $\mathrm{Ti}$ in quartz and those estimated from garnet and biotite core compositions (Tables 1 and 2). Garnet and biotite rim compositions however have recorded cooling to significantly lower temperatures, which was not recorded 
Table 1. Titanium content and calculated TitaniQ temperatures for each analytical spot, with the average values shown for each sample.

\begin{tabular}{|c|c|c|c|c|c|}
\hline \multirow{2}{*}{$\begin{array}{l}\text { Sample-crystal } \\
\text { number }\end{array}$} & \multirow[t]{2}{*}{ Lithology } & \multirow{2}{*}{$\begin{array}{c}\mathrm{Ti} \\
(\mathrm{ppm})\end{array}$} & \multicolumn{3}{|c|}{$T\left({ }^{\circ} \mathrm{C}\right), P=650 \mathrm{Mpa}$} \\
\hline & & & Thomas et al. (2010) & Wark and Watson (2006) & Huang and Audétat (2012) \\
\hline ar1296pt01 & Migmatitic granulite & 92 & 667 & 737 & 831 \\
\hline ar1296pt01 & Migmatitic granulite & 98 & 673 & 745 & 840 \\
\hline $\operatorname{ar} 1296 \mathrm{pt} 01$ & Migmatitic granulite & 89 & 663 & 733 & 827 \\
\hline $\operatorname{ar} 1296 \mathrm{pt} 01$ & Migmatitic granulite & 27 & 557 & 611 & 692 \\
\hline $\operatorname{ar} 1296 \mathrm{pt} 01$ & Migmatitic granulite & 83 & 656 & 725 & 818 \\
\hline $\operatorname{ar} 1296 \mathrm{pt} 02$ & Migmatitic granulite & 112 & 687 & 761 & 858 \\
\hline $\operatorname{ar} 1296 \mathrm{pt} 02$ & Migmatitic granulite & 110 & 685 & 759 & 855 \\
\hline $\operatorname{ar} 1296 \mathrm{pt} 02$ & Migmatitic granulite & 109 & 684 & 758 & 854 \\
\hline $\operatorname{ar} 1296 \mathrm{pt} 02$ & Migmatitic granulite & 118 & 693 & 767 & 865 \\
\hline ar1296pt02 & Migmatitic granulite & 136 & 708 & 785 & 885 \\
\hline $\operatorname{ar} 1296 \mathrm{pt} 02$ & Migmatitic granulite & 141 & 712 & 790 & 890 \\
\hline $\operatorname{ar} 1296 \mathrm{pt} 03$ & Migmatitic granulite & 91 & 665 & 736 & 830 \\
\hline $\operatorname{ar} 1296 \mathrm{pt} 03$ & Migmatitic granulite & 107 & 682 & 755 & 851 \\
\hline $\operatorname{ar} 1296 \mathrm{pt} 03$ & Migmatitic granulite & 156 & 723 & 804 & 905 \\
\hline $\operatorname{ar} 1296 \mathrm{pt} 03$ & Migmatitic granulite & 265 & 788 & 879 & 989 \\
\hline $\operatorname{ar} 1296 \mathrm{pt} 03$ & Migmatitic granulite & 103 & 678 & 751 & 846 \\
\hline ar1296pt04 & Migmatitic granulite & 97 & 672 & 744 & 838 \\
\hline ar1296pt04 & Migmatitic granulite & 91 & 665 & 736 & 830 \\
\hline ar1296pt04 & Migmatitic granulite & 101 & 676 & 748 & 844 \\
\hline ar1296pt04 & Migmatitic granulite & 122 & 696 & 772 & 869 \\
\hline ar1296pt04 & Migmatitic granulite & 103 & 678 & 751 & 846 \\
\hline ar1296pt04 & Migmatitic granulite & 88 & 662 & 732 & 825 \\
\hline ar1296pt05 & Migmatitic granulite & 104 & 679 & 752 & 847 \\
\hline ar1296pt05 & Migmatitic granulite & 112 & 687 & 761 & 858 \\
\hline $\operatorname{ar} 1296 \mathrm{pt} 05$ & Migmatitic granulite & 125 & 699 & 775 & 873 \\
\hline ar1296pt05 & Migmatitic granulite & 124 & 698 & 774 & 872 \\
\hline ar1296 (average) & Migmatitic granulite & 112 & $687 \pm 10$ & $755 \pm 16$ & $842 \pm 20$ \\
\hline $\operatorname{ar1299pt01}$ & Diatexite & 117 & 692 & 766 & 863 \\
\hline $\operatorname{ar} 1299 \mathrm{pt} 01$ & Diatexite & 142 & 713 & 791 & 891 \\
\hline $\operatorname{ar} 1299 \mathrm{pt} 01$ & Diatexite & 136 & 708 & 785 & 885 \\
\hline $\operatorname{ar} 1299 \mathrm{pt} 01$ & Diatexite & 123 & 697 & 773 & 870 \\
\hline $\operatorname{ar} 1299 \mathrm{pt} 01$ & Diatexite & 128 & 701 & 778 & 876 \\
\hline $\operatorname{ar} 1299 \mathrm{pt} 01$ & Diatexite & 126 & 700 & 776 & 874 \\
\hline $\operatorname{ar} 1299 \mathrm{pt} 01$ & Diatexite & 132 & 705 & 782 & 880 \\
\hline $\operatorname{ar} 1299 \mathrm{pt} 01$ & Diatexite & 153 & 721 & 801 & 902 \\
\hline $\operatorname{ar} 1299 \mathrm{pt} 01$ & Diatexite & 136 & 708 & 785 & 885 \\
\hline $\operatorname{ar} 1299 \mathrm{pt} 01$ & Diatexite & 120 & 694 & 770 & 867 \\
\hline $\operatorname{ar} 1299 \mathrm{pt} 01$ & Diatexite & 132 & 705 & 782 & 880 \\
\hline ar1299pt01 & Diatexite & 130 & 703 & 780 & 878 \\
\hline $\operatorname{ar} 1299 \mathrm{pt} 01$ & Diatexite & 124 & 698 & 774 & 872 \\
\hline ar1299pt01 & Diatexite & 130 & 703 & 780 & 878 \\
\hline $\operatorname{ar} 1299 \mathrm{pt} 01$ & Diatexite & 119 & 693 & 769 & 866 \\
\hline ar1299pt01 & Diatexite & 132 & 705 & 782 & 880 \\
\hline ar1299pt01 & Diatexite & 115 & 690 & 764 & 861 \\
\hline ar1299pt01 & Diatexite & 114 & 689 & 763 & 860 \\
\hline ar1299pt03 & Diatexite & 117 & 692 & 766 & 863 \\
\hline $\operatorname{ar} 1299 \mathrm{pt} 03$ & Diatexite & 125 & 699 & 775 & 873 \\
\hline ar1299pt03 & Diatexite & 118 & 693 & 767 & 865 \\
\hline ar1299pt03 & Diatexite & 118 & 693 & 767 & 865 \\
\hline ar1299pt03 & Diatexite & 132 & 705 & 782 & 880 \\
\hline ar1299pt03 & Diatexite & 127 & 700 & 777 & 875 \\
\hline $\operatorname{ar} 1299 \mathrm{pt} 03$ & Diatexite & 149 & 718 & 797 & 898 \\
\hline $\operatorname{ar} 1299 \mathrm{pt} 03$ & Diatexite & 140 & 711 & 789 & 889 \\
\hline
\end{tabular}


Table 1. Continued.

\begin{tabular}{|c|c|c|c|c|c|}
\hline \multirow{2}{*}{$\begin{array}{l}\text { Sample-crystal } \\
\text { number }\end{array}$} & \multirow[t]{2}{*}{ Lithology } & \multirow{2}{*}{$\begin{array}{c}\mathrm{Ti} \\
(\mathrm{ppm})\end{array}$} & \multicolumn{3}{|c|}{$T\left({ }^{\circ} \mathrm{C}\right), P=650 \mathrm{Mpa}$} \\
\hline & & & Thomas et al. (2010) & Wark and Watson (2006) & Huang and Audétat (2012) \\
\hline $\operatorname{ar} 1299 \mathrm{pt} 03$ & Diatexite & 136 & 708 & 785 & 885 \\
\hline $\operatorname{ar} 1299 \mathrm{pt} 03$ & Diatexite & 124 & 698 & 774 & 872 \\
\hline $\operatorname{ar} 1299 \mathrm{pt} 03$ & Diatexite & 138 & 710 & 787 & 887 \\
\hline $\operatorname{ar} 1299 \mathrm{pt} 03$ & Diatexite & 108 & 693 & 757 & 853 \\
\hline $\operatorname{ar} 1299 \mathrm{pt} 03$ & Diatexite & 122 & 696 & 772 & 869 \\
\hline $\operatorname{ar} 1299 \mathrm{pt} 03$ & Diatexite & 144 & 714 & 793 & 893 \\
\hline $\operatorname{ar} 1299 \mathrm{pt} 03$ & Diatexite & 135 & 707 & 785 & 884 \\
\hline $\operatorname{ar} 1299 \mathrm{pt} 03$ & Diatexite & 138 & 710 & 787 & 887 \\
\hline ar1299pt03 & Diatexite & 123 & 697 & 773 & 870 \\
\hline ar1299pt04 & Diatexite & 122 & 696 & 772 & 869 \\
\hline $\operatorname{ar} 1299 \mathrm{pt} 04$ & Diatexite & 119 & 693 & 769 & 866 \\
\hline ar1299pt04 & Diatexite & 107 & 682 & 755 & 851 \\
\hline ar1299pt04 & Diatexite & 139 & 710 & 788 & 888 \\
\hline ar1299pt04 & Diatexite & 139 & 710 & 788 & 888 \\
\hline ar1299pt04 & Diatexite & 147 & 717 & 796 & 896 \\
\hline ar1299pt04 & Diatexite & 162 & 728 & 809 & 910 \\
\hline ar1299pt04 & Diatexite & 141 & 712 & 790 & 890 \\
\hline ar1299pt04 & Diatexite & 156 & 723 & 804 & 905 \\
\hline ar1299pt04 & Diatexite & 135 & 707 & 785 & 884 \\
\hline ar1299pt04 & Diatexite & 150 & 719 & 798 & 899 \\
\hline ar1299pt04 & Diatexite & 136 & 708 & 785 & 885 \\
\hline ar1299pt04 & Diatexite & 134 & 706 & 784 & 883 \\
\hline ar1299pt04 & Diatexite & 134 & 706 & 784 & 883 \\
\hline ar1299pt04 & Diatexite & 112 & 687 & 761 & 858 \\
\hline ar1299pt04 & Diatexite & 122 & 696 & 772 & 869 \\
\hline ar1299pt04 & Diatexite & 143 & 714 & 792 & 892 \\
\hline ar1299pt04 & Diatexite & 146 & 716 & 795 & 895 \\
\hline ar1299pt04 & Diatexite & 144 & 714 & 793 & 893 \\
\hline ar1299pt04 & Diatexite & 152 & 721 & 800 & 901 \\
\hline ar1299pt04 & Diatexite & 141 & 712 & 790 & 890 \\
\hline ar1299pt05 & Diatexite & 123 & 697 & 773 & 870 \\
\hline ar1299pt05 & Diatexite & 115 & 690 & 764 & 861 \\
\hline ar1299pt05 & Diatexite & 124 & 698 & 774 & 872 \\
\hline ar1299pt05 & Diatexite & 127 & 700 & 777 & 875 \\
\hline ar1299pt05 & Diatexite & 124 & 698 & 774 & 872 \\
\hline ar1299pt05 & Diatexite & 130 & 703 & 780 & 878 \\
\hline ar1299pt05 & Diatexite & 121 & 695 & 771 & 868 \\
\hline ar1299pt05 & Diatexite & 126 & 700 & 776 & 874 \\
\hline ar1299pt05 & Diatexite & 140 & 711 & 789 & 889 \\
\hline ar1299pt05 & Diatexite & 118 & 693 & 767 & 865 \\
\hline ar1299pt05 & Diatexite & 109 & 684 & 758 & 854 \\
\hline ar1299 (average) & Diatexite & 130 & $703 \pm 10$ & $780 \pm 16$ & $868 \pm 20$ \\
\hline ar1258pt01 & Diatexite & 82 & 655 & 724 & 816 \\
\hline $\operatorname{ar} 1258 \mathrm{pt} 01$ & Diatexite & 102 & 677 & 750 & 845 \\
\hline $\operatorname{ar} 1258 \mathrm{pt} 01$ & Diatexite & 79 & 651 & 720 & 812 \\
\hline $\operatorname{ar} 1258 \mathrm{pt} 01$ & Diatexite & 75 & 646 & 714 & 805 \\
\hline ar1258pt01 & Diatexite & 82 & 655 & 724 & 816 \\
\hline ar1258pt01 & Diatexite & 88 & 662 & 732 & 825 \\
\hline $\operatorname{ar} 1258 \mathrm{pt} 01$ & Diatexite & 85 & 659 & 728 & 821 \\
\hline $\operatorname{ar} 1258 \mathrm{pt} 01$ & Diatexite & 143 & 714 & 792 & 892 \\
\hline $\operatorname{ar} 1258 \mathrm{pt} 01$ & Diatexite & 92 & 667 & 737 & 831 \\
\hline $\operatorname{ar} 1258 \mathrm{pt} 01$ & Diatexite & 112 & 687 & 761 & 858 \\
\hline $\operatorname{ar} 1258 \mathrm{pt} 01$ & Diatexite & 93 & 668 & 739 & 833 \\
\hline
\end{tabular}


Table 1. Continued.

\begin{tabular}{|c|c|c|c|c|c|}
\hline \multirow{2}{*}{$\begin{array}{l}\text { Sample-crystal } \\
\text { number }\end{array}$} & \multirow[t]{2}{*}{ Lithology } & \multirow{2}{*}{$\begin{array}{c}\mathrm{Ti} \\
(\mathrm{ppm})\end{array}$} & \multicolumn{3}{|c|}{$T\left({ }^{\circ} \mathrm{C}\right), P=650 \mathrm{Mpa}$} \\
\hline & & & Thomas et al. (2010) & Wark and Watson (2006) & Huang and Audétat (2012) \\
\hline $\operatorname{ar} 1258 \mathrm{pt} 01$ & Diatexite & 88 & 662 & 732 & 825 \\
\hline $\operatorname{ar} 1258 \mathrm{pt} 01$ & Diatexite & 92 & 667 & 737 & 831 \\
\hline $\operatorname{ar} 1258 \mathrm{pt} 01$ & Diatexite & 96 & 671 & 742 & 837 \\
\hline $\operatorname{ar} 1258 \mathrm{pt} 01$ & Diatexite & 87 & 661 & 731 & 824 \\
\hline $\operatorname{ar} 1258 \mathrm{pt} 01$ & Diatexite & 126 & 700 & 776 & 874 \\
\hline $\operatorname{ar} 1258 \mathrm{pt} 01$ & Diatexite & 85 & 659 & 728 & 821 \\
\hline ar1258pt01 & Diatexite & 101 & 676 & 748 & 844 \\
\hline ar1258pt01 & Diatexite & 99 & 674 & 746 & 841 \\
\hline $\operatorname{ar} 1258 \mathrm{pt} 01$ & Diatexite & 86 & 660 & 729 & 822 \\
\hline $\operatorname{ar} 1258 \mathrm{pt} 01$ & Diatexite & 88 & 662 & 732 & 825 \\
\hline $\operatorname{ar} 1258 \mathrm{pt} 01$ & Diatexite & 86 & 660 & 729 & 822 \\
\hline ar1258pt01 & Diatexite & 82 & 655 & 724 & 816 \\
\hline $\operatorname{ar} 1258 \mathrm{pt} 01$ & Diatexite & 78 & 650 & 718 & 810 \\
\hline $\operatorname{ar} 1258 \mathrm{pt} 01$ & Diatexite & 77 & 649 & 717 & 808 \\
\hline $\operatorname{ar} 1258 \mathrm{pt} 01$ & Diatexite & 83 & 656 & 725 & 818 \\
\hline $\operatorname{ar} 1258 \mathrm{pt} 01$ & Diatexite & 103 & 678 & 751 & 846 \\
\hline ar1258pt02 & Diatexite & 97 & 672 & 744 & 838 \\
\hline ar1258pt02 & Diatexite & 104 & 679 & 752 & 847 \\
\hline ar1258pt02 & Diatexite & 88 & 662 & 732 & 825 \\
\hline $\operatorname{ar} 1258 \mathrm{pt} 02$ & Diatexite & 99 & 674 & 746 & 841 \\
\hline $\operatorname{ar} 1258 \mathrm{pt} 02$ & Diatexite & 112 & 687 & 761 & 858 \\
\hline ar1258pt03 & Diatexite & 89 & 663 & 733 & 827 \\
\hline ar1258pt03 & Diatexite & 97 & 672 & 744 & 838 \\
\hline ar1258pt03 & Diatexite & 147 & 717 & 796 & 896 \\
\hline ar1258pt03 & Diatexite & 101 & 676 & 748 & 844 \\
\hline ar $1258 \mathrm{pt} 03$ & Diatexite & 77 & 649 & 717 & 808 \\
\hline ar1258pt03 & Diatexite & 87 & 661 & 731 & 824 \\
\hline ar1258pt03 & Diatexite & 108 & 693 & 757 & 853 \\
\hline ar1258pt03 & Diatexite & 89 & 663 & 733 & 827 \\
\hline ar1258pt03 & Diatexite & 99 & 674 & 746 & 841 \\
\hline ar1258pt03 & Diatexite & 121 & 695 & 771 & 868 \\
\hline ar1258pt03 & Diatexite & 84 & 657 & 727 & 819 \\
\hline ar1258pt03 & Diatexite & 100 & 675 & 747 & 842 \\
\hline ar1258pt03 & Diatexite & 93 & 668 & 739 & 833 \\
\hline ar1258pt04 & Diatexite & 96 & 671 & 742 & 837 \\
\hline ar1258pt04 & Diatexite & 97 & 672 & 744 & 838 \\
\hline ar1258pt04 & Diatexite & 96 & 671 & 742 & 837 \\
\hline ar1258pt04 & Diatexite & 88 & 662 & 732 & 825 \\
\hline ar1258pt04 & Diatexite & 90 & 664 & 735 & 828 \\
\hline ar1258pt04 & Diatexite & 89 & 663 & 733 & 827 \\
\hline ar1258pt04 & Diatexite & 91 & 665 & 736 & 830 \\
\hline ar1258pt04 & Diatexite & 108 & 693 & 757 & 853 \\
\hline ar1258pt04 & Diatexite & 95 & 670 & 741 & 835 \\
\hline ar1258pt04 & Diatexite & 101 & 676 & 748 & 844 \\
\hline ar1258pt04 & Diatexite & 97 & 672 & 744 & 838 \\
\hline ar1258pt04 & Diatexite & 112 & 687 & 761 & 858 \\
\hline ar1258pt04 & Diatexite & 102 & 677 & 750 & 845 \\
\hline ar1258pt05 & Diatexite & 131 & 704 & 781 & 879 \\
\hline ar1258pt05 & Diatexite & 98 & 673 & 745 & 840 \\
\hline ar1258pt05 & Diatexite & 91 & 665 & 736 & 830 \\
\hline ar1258pt05 & Diatexite & 92 & 667 & 737 & 831 \\
\hline ar1258pt05 & Diatexite & 102 & 677 & 750 & 845 \\
\hline ar1258pt05 & Diatexite & 94 & 669 & 740 & 834 \\
\hline ar1258pt05 & Diatexite & 103 & 678 & 751 & 846 \\
\hline
\end{tabular}


Table 1. Continued.

\begin{tabular}{|c|c|c|c|c|c|}
\hline \multirow{2}{*}{$\begin{array}{l}\text { Sample-crystal } \\
\text { number }\end{array}$} & \multirow[t]{2}{*}{ Lithology } & \multirow{2}{*}{$\begin{array}{c}\mathrm{Ti} \\
(\mathrm{ppm})\end{array}$} & \multicolumn{3}{|c|}{$T\left({ }^{\circ} \mathrm{C}\right), P=650 \mathrm{Mpa}$} \\
\hline & & & Thomas et al. (2010) & Wark and Watson (2006) & Huang and Audétat (2012) \\
\hline ar1258pt05 & Diatexite & 111 & 686 & 760 & 856 \\
\hline $\operatorname{ar} 1258 \mathrm{pt} 05$ & Diatexite & 120 & 694 & 770 & 867 \\
\hline ar1258pt05 & Diatexite & 181 & 741 & 824 & 927 \\
\hline ar1258pt05 & Diatexite & 92 & 667 & 737 & 831 \\
\hline ar1258pt05 & Diatexite & 93 & 668 & 739 & 833 \\
\hline $\operatorname{ar} 1258 \mathrm{pt} 05$ & Diatexite & 202 & 754 & 839 & 944 \\
\hline ar1258pt05 & Diatexite & 100 & 675 & 747 & 842 \\
\hline ar1258pt05 & Diatexite & 116 & 691 & 765 & 862 \\
\hline $\operatorname{ar} 1258 \mathrm{pt} 05$ & Diatexite & 99 & 674 & 746 & 841 \\
\hline $\operatorname{ar} 1258 \mathrm{pt} 05$ & Diatexite & 97 & 672 & 744 & 838 \\
\hline $\operatorname{ar} 1258 \mathrm{pt} 05$ & Diatexite & 137 & 709 & 786 & 886 \\
\hline ar1258pt05 & Diatexite & 101 & 676 & 748 & 844 \\
\hline ar1258pt05 & Diatexite & 98 & 673 & 745 & 840 \\
\hline ar1258pt05 & Diatexite & 123 & 697 & 773 & 870 \\
\hline ar1258pt05 & Diatexite & 106 & 681 & 754 & 850 \\
\hline ar1258pt05 & Diatexite & 108 & 693 & 757 & 853 \\
\hline ar1258pt05 & Diatexite & 95 & 670 & 741 & 835 \\
\hline ar1258pt05 & Diatexite & 109 & 684 & 758 & 854 \\
\hline ar1258pt05 & Diatexite & 129 & 702 & 779 & 877 \\
\hline ar1258pt05 & Diatexite & 100 & 675 & 747 & 842 \\
\hline $\operatorname{ar} 1258 \mathrm{pt} 05$ & Diatexite & 85 & 659 & 728 & 821 \\
\hline ar1258 (average) & Diatexite & 101 & $676 \pm 10$ & $747 \pm 15$ & $848 \pm 20$ \\
\hline $\operatorname{ar} 1326 \mathrm{pt} 02$ & Metatexite & 112 & 687 & 761 & 858 \\
\hline $\operatorname{ar} 1326 \mathrm{pt} 02$ & Metatexite & 157 & 724 & 804 & 906 \\
\hline ar1326pt02 & Metatexite & 157 & 724 & 804 & 906 \\
\hline $\operatorname{ar} 1326 \mathrm{pt} 02$ & Metatexite & 155 & 723 & 803 & 904 \\
\hline $\operatorname{ar} 1326 \mathrm{pt} 02$ & Metatexite & 154 & 722 & 802 & 903 \\
\hline $\operatorname{ar} 1326 \mathrm{pt} 02$ & Metatexite & 103 & 678 & 751 & 846 \\
\hline $\operatorname{ar} 1326 \mathrm{pt} 02$ & Metatexite & 15 & 512 & 561 & 637 \\
\hline $\operatorname{ar} 1326 \mathrm{pt} 02$ & Metatexite & 157 & 724 & 804 & 906 \\
\hline ar1326pt02 & Metatexite & 150 & 719 & 798 & 899 \\
\hline ar1326pt02 & Metatexite & 138 & 710 & 787 & 887 \\
\hline $\operatorname{ar} 1326 \mathrm{pt} 02$ & Metatexite & 137 & 709 & 786 & 886 \\
\hline ar1326pt03 & Metatexite & 158 & 725 & 805 & 907 \\
\hline $\operatorname{ar} 1326 \mathrm{pt} 03$ & Metatexite & 152 & 721 & 800 & 901 \\
\hline $\operatorname{ar} 1326 \mathrm{pt} 03$ & Metatexite & 145 & 715 & 794 & 894 \\
\hline ar1326pt03 & Metatexite & 156 & 723 & 804 & 905 \\
\hline $\operatorname{ar} 1326 \mathrm{pt} 03$ & Metatexite & 143 & 714 & 792 & 892 \\
\hline ar1326pt03 & Metatexite & 129 & 702 & 779 & 877 \\
\hline $\operatorname{ar} 1326 \mathrm{pt} 03$ & Metatexite & 124 & 698 & 774 & 872 \\
\hline $\operatorname{ar} 1326 \mathrm{pt} 03$ & Metatexite & 110 & 685 & 759 & 855 \\
\hline ar1326pt03 & Metatexite & 127 & 700 & 777 & 875 \\
\hline $\operatorname{ar} 1326 \mathrm{pt} 03$ & Metatexite & 128 & 701 & 778 & 876 \\
\hline $\operatorname{ar} 1326 \mathrm{pt} 03$ & Metatexite & 137 & 709 & 786 & 886 \\
\hline $\operatorname{ar} 1326 \mathrm{pt} 03$ & Metatexite & 148 & 717 & 797 & 897 \\
\hline ar1326pt03 & Metatexite & 157 & 724 & 804 & 906 \\
\hline ar1326pt03 & Metatexite & 136 & 708 & 785 & 885 \\
\hline $\operatorname{ar} 1326 \mathrm{pt} 03$ & Metatexite & 131 & 704 & 781 & 879 \\
\hline $\operatorname{ar} 1326 \mathrm{pt} 03$ & Metatexite & 123 & 697 & 773 & 870 \\
\hline $\operatorname{ar} 1326 \mathrm{pt} 03$ & Metatexite & 135 & 707 & 785 & 884 \\
\hline ar1326pt03 & Metatexite & 128 & 701 & 778 & 876 \\
\hline $\operatorname{ar} 1326 \mathrm{pt} 06$ & Metatexite & 131 & 704 & 781 & 879 \\
\hline ar1326pt06 & Metatexite & 152 & 721 & 800 & 901 \\
\hline
\end{tabular}


Table 1. Continued.

\begin{tabular}{|c|c|c|c|c|c|}
\hline \multirow{2}{*}{$\begin{array}{l}\text { Sample-crystal } \\
\text { number }\end{array}$} & \multirow[t]{2}{*}{ Lithology } & \multirow{2}{*}{$\begin{array}{c}\mathrm{Ti} \\
(\mathrm{ppm})\end{array}$} & \multicolumn{3}{|c|}{$T\left({ }^{\circ} \mathrm{C}\right), P=650 \mathrm{Mpa}$} \\
\hline & & & Thomas et al. (2010) & Wark and Watson (2006) & Huang and Audétat (2012) \\
\hline ar1326pt06 & Metatexite & 148 & 717 & 797 & 897 \\
\hline $\operatorname{ar} 1326 \mathrm{pt} 06$ & Metatexite & 144 & 714 & 793 & 893 \\
\hline $\operatorname{ar} 1326 \mathrm{pt} 06$ & Metatexite & 144 & 714 & 793 & 893 \\
\hline $\operatorname{ar} 1326 \mathrm{pt} 06$ & Metatexite & 146 & 716 & 795 & 895 \\
\hline $\operatorname{ar} 1326 \mathrm{pt} 06$ & Metatexite & 157 & 724 & 804 & 906 \\
\hline ar1326pt06 & Metatexite & 162 & 728 & 809 & 910 \\
\hline $\operatorname{ar} 1326 \mathrm{pt} 06$ & Metatexite & 160 & 726 & 807 & 908 \\
\hline $\operatorname{ar} 1326 \mathrm{pt} 06$ & Metatexite & 154 & 722 & 802 & 903 \\
\hline $\operatorname{ar} 1326 \mathrm{pt} 06$ & Metatexite & 160 & 726 & 807 & 908 \\
\hline ar1326pt06 & Metatexite & 157 & 724 & 804 & 906 \\
\hline $\operatorname{ar} 1326 \mathrm{pt} 06$ & Metatexite & 145 & 715 & 794 & 894 \\
\hline $\operatorname{ar} 1326 \mathrm{pt} 06$ & Metatexite & 145 & 715 & 794 & 894 \\
\hline $\operatorname{ar} 1326 \mathrm{pt} 06$ & Metatexite & 140 & 711 & 789 & 889 \\
\hline ar1326pt06 & Metatexite & 123 & 697 & 773 & 870 \\
\hline ar1326pt06 & Metatexite & 151 & 720 & 799 & 900 \\
\hline ar1326pt06 & Metatexite & 152 & 721 & 800 & 901 \\
\hline ar1326pt06 & Metatexite & 154 & 722 & 802 & 903 \\
\hline ar1326pt06 & Metatexite & 148 & 717 & 797 & 897 \\
\hline ar1326pt06 & Metatexite & 142 & 713 & 791 & 891 \\
\hline ar1326pt06 & Metatexite & 156 & 723 & 804 & 905 \\
\hline ar1326pt06 & Metatexite & 147 & 717 & 796 & 896 \\
\hline ar1326pt06 & Metatexite & 124 & 698 & 774 & 872 \\
\hline $\operatorname{ar} 1326 \mathrm{pt} 07$ & Metatexite & 153 & 721 & 801 & 902 \\
\hline $\operatorname{ar} 1326 \mathrm{pt} 07$ & Metatexite & 160 & 726 & 807 & 908 \\
\hline $\operatorname{ar} 1326 \mathrm{pt} 07$ & Metatexite & 160 & 726 & 807 & 908 \\
\hline ar1326pt07 & Metatexite & 154 & 722 & 802 & 903 \\
\hline ar1326pt07 & Metatexite & 166 & 731 & 812 & 914 \\
\hline ar1326pt07 & Metatexite & 162 & 728 & 809 & 910 \\
\hline $\operatorname{ar} 1326 \mathrm{pt} 07$ & Metatexite & 170 & 733 & 815 & 917 \\
\hline $\operatorname{ar} 1326 \mathrm{pt} 07$ & Metatexite & 171 & 733 & 816 & 918 \\
\hline ar1326pt07 & Metatexite & 165 & 730 & 811 & 913 \\
\hline $\operatorname{ar} 1326 \mathrm{pt} 07$ & Metatexite & 161 & 727 & 808 & 909 \\
\hline ar1326pt07 & Metatexite & 150 & 719 & 798 & 899 \\
\hline $\operatorname{ar} 1326 \mathrm{pt} 07$ & Metatexite & 159 & 726 & 806 & 907 \\
\hline ar1326pt07 & Metatexite & 160 & 726 & 807 & 908 \\
\hline ar1326pt07 & Metatexite & 162 & 728 & 809 & 910 \\
\hline ar1326pt07 & Metatexite & 161 & 727 & 808 & 909 \\
\hline ar1326 (average) & Metatexite & 145 & $715 \pm 10$ & $792 \pm 17$ & $881 \pm 20$ \\
\hline $\operatorname{ar} 1083 \mathrm{pt} 01$ & Migmatitic kinzigites & 139 & 710 & 788 & 888 \\
\hline $\operatorname{ar} 1083 \mathrm{pt} 01$ & Migmatitic kinzigites & 145 & 715 & 794 & 894 \\
\hline $\operatorname{ar} 1083 \mathrm{pt} 01$ & Migmatitic kinzigites & 150 & 719 & 798 & 899 \\
\hline $\operatorname{ar} 1083 \mathrm{pt} 01$ & Migmatitic kinzigites & 153 & 721 & 801 & 902 \\
\hline $\operatorname{ar} 1083 \mathrm{pt} 01$ & Migmatitic kinzigites & 152 & 721 & 800 & 901 \\
\hline $\operatorname{ar} 1083 \mathrm{pt} 01$ & Migmatitic kinzigites & 152 & 721 & 800 & 901 \\
\hline $\operatorname{ar} 1083 \mathrm{pt} 01$ & Migmatitic kinzigites & 145 & 715 & 794 & 894 \\
\hline $\operatorname{ar} 1083 \mathrm{pt} 03$ & Migmatitic kinzigites & 134 & 706 & 784 & 883 \\
\hline $\operatorname{ar} 1083 \mathrm{pt} 03$ & Migmatitic kinzigites & 136 & 708 & 785 & 885 \\
\hline $\operatorname{ar} 1083 \mathrm{pt} 03$ & Migmatitic kinzigites & 144 & 714 & 793 & 893 \\
\hline $\operatorname{ar} 1083 \mathrm{pt} 03$ & Migmatitic kinzigites & 135 & 707 & 785 & 884 \\
\hline $\operatorname{ar} 1083 \mathrm{pt} 03$ & Migmatitic kinzigites & 134 & 706 & 784 & 883 \\
\hline $\operatorname{ar} 1083 \mathrm{pt} 03$ & Migmatitic kinzigites & 136 & 707 & 785 & 885 \\
\hline $\operatorname{ar} 1083 \mathrm{pt} 04$ & Migmatitic kinzigites & 126 & 700 & 776 & 874 \\
\hline $\operatorname{ar} 1083 \mathrm{pt} 04$ & Migmatitic kinzigites & 142 & 713 & 791 & 891 \\
\hline
\end{tabular}


Table 1. Continued.

\begin{tabular}{|c|c|c|c|c|c|}
\hline \multirow{2}{*}{$\begin{array}{l}\text { Sample-crystal } \\
\text { number }\end{array}$} & \multirow[t]{2}{*}{ Lithology } & \multirow{2}{*}{$\begin{array}{c}\mathrm{Ti} \\
(\mathrm{ppm})\end{array}$} & \multicolumn{3}{|c|}{$T\left({ }^{\circ} \mathrm{C}\right), P=650 \mathrm{Mpa}$} \\
\hline & & & Thomas et al. (2010) & Wark and Watson (2006) & Huang and Audétat (2012) \\
\hline ar1083pt04 & Migmatitic kinzigites & 144 & 714 & 793 & 893 \\
\hline $\operatorname{ar} 1083 \mathrm{pt} 04$ & Migmatitic kinzigites & 144 & 714 & 793 & 893 \\
\hline $\operatorname{ar} 1083 \mathrm{pt} 04$ & Migmatitic kinzigites & 138 & 710 & 787 & 887 \\
\hline $\operatorname{ar} 1083 \mathrm{pt} 04$ & Migmatitic kinzigites & 134 & 706 & 784 & 883 \\
\hline ar1083pt04 & Migmatitic kinzigites & 127 & 700 & 777 & 875 \\
\hline $\operatorname{ar} 1083 \mathrm{pt} 04$ & Migmatitic kinzigites & 127 & 700 & 777 & 875 \\
\hline $\operatorname{ar} 1083 \mathrm{pt} 04$ & Migmatitic kinzigites & 134 & 706 & 784 & 883 \\
\hline $\operatorname{ar} 1083 \mathrm{pt} 04$ & Migmatitic kinzigites & 148 & 717 & 797 & 897 \\
\hline ar1083pt04 & Migmatitic kinzigites & 127 & 700 & 777 & 875 \\
\hline $\operatorname{ar} 1083 \mathrm{pt} 04$ & Migmatitic kinzigites & 112 & 687 & 761 & 858 \\
\hline ar1083 (average) & Migmatitic kinzigites & 138 & $710 \pm 10$ & $787 \pm 17$ & $877 \pm 20$ \\
\hline $\operatorname{ar} 949 p t 01$ & Migmatitic kinzigites & 154 & 722 & 802 & 903 \\
\hline $\operatorname{ar} 949 \mathrm{pt} 01$ & Migmatitic kinzigites & 167 & 731 & 813 & 915 \\
\hline $\operatorname{ar} 949 \mathrm{pt} 01$ & Migmatitic kinzigites & 167 & 731 & 813 & 915 \\
\hline $\operatorname{ar} 949 p t 01$ & Migmatitic kinzigites & 170 & 733 & 815 & 917 \\
\hline $\operatorname{ar} 949 p t 01$ & Migmatitic kinzigites & 171 & 733 & 816 & 918 \\
\hline $\operatorname{ar} 949 \mathrm{pt} 01$ & Migmatitic kinzigites & 175 & 737 & 819 & 922 \\
\hline ar949pt01 & Migmatitic kinzigites & 175 & 737 & 819 & 922 \\
\hline $\operatorname{ar} 949 \mathrm{pt} 01$ & Migmatitic kinzigites & 161 & 727 & 808 & 909 \\
\hline $\operatorname{ar} 949 \mathrm{pt} 01$ & Migmatitic kinzigites & 156 & 723 & 804 & 905 \\
\hline $\operatorname{ar} 949 \mathrm{pt} 01$ & Migmatitic kinzigites & 155 & 723 & 803 & 904 \\
\hline $\operatorname{ar} 949 \mathrm{pt} 01$ & Migmatitic kinzigites & 133 & 706 & 783 & 881 \\
\hline $\operatorname{ar} 949 p t 01$ & Migmatitic kinzigites & 152 & 721 & 800 & 901 \\
\hline ar949pt01 & Migmatitic kinzigites & 159 & 726 & 806 & 907 \\
\hline $\operatorname{ar} 949 \mathrm{pt} 01$ & Migmatitic kinzigites & 168 & 732 & 814 & 916 \\
\hline $\operatorname{ar} 949 \mathrm{pt} 01$ & Migmatitic kinzigites & 181 & 741 & 824 & 927 \\
\hline $\operatorname{ar} 949 p t 01$ & Migmatitic kinzigites & 179 & 739 & 822 & 925 \\
\hline $\operatorname{ar} 949 \mathrm{pt} 01$ & Migmatitic kinzigites & 163 & 729 & 809 & 911 \\
\hline $\operatorname{ar} 949 \mathrm{pt} 01$ & Migmatitic kinzigites & 169 & 733 & 814 & 917 \\
\hline $\operatorname{ar} 949 \mathrm{pt} 01$ & Migmatitic kinzigites & 156 & 723 & 804 & 905 \\
\hline $\operatorname{ar} 949 \mathrm{pt} 01$ & Migmatitic kinzigites & 147 & 717 & 796 & 896 \\
\hline $\operatorname{ar} 949 \mathrm{pt} 02$ & Migmatitic kinzigites & 140 & 711 & 789 & 889 \\
\hline $\operatorname{ar} 949 \mathrm{pt} 02$ & Migmatitic kinzigites & 139 & 710 & 788 & 888 \\
\hline $\operatorname{ar} 949 \mathrm{pt} 02$ & Migmatitic kinzigites & 109 & 684 & 758 & 854 \\
\hline ar949pt02 & Migmatitic kinzigites & 114 & 689 & 763 & 860 \\
\hline $\operatorname{ar} 949 \mathrm{pt} 02$ & Migmatitic kinzigites & 133 & 706 & 783 & 881 \\
\hline $\operatorname{ar} 949 \mathrm{pt} 03$ & Migmatitic kinzigites & 107 & 682 & 755 & 851 \\
\hline $\operatorname{ar} 949 \mathrm{pt} 03$ & Migmatitic kinzigites & 126 & 700 & 776 & 874 \\
\hline $\operatorname{ar} 949 \mathrm{pt} 03$ & Migmatitic kinzigites & 125 & 699 & 775 & 873 \\
\hline ar949pt03 & Migmatitic kinzigites & 122 & 696 & 772 & 869 \\
\hline $\operatorname{ar} 949 \mathrm{pt} 03$ & Migmatitic kinzigites & 132 & 705 & 782 & 880 \\
\hline $\operatorname{ar} 949 \mathrm{pt} 03$ & Migmatitic kinzigites & 111 & 686 & 760 & 856 \\
\hline $\operatorname{ar} 949 \mathrm{pt} 03$ & Migmatitic kinzigites & 111 & 686 & 760 & 856 \\
\hline $\operatorname{ar} 949 \mathrm{pt} 03$ & Migmatitic kinzigites & 127 & 700 & 777 & 875 \\
\hline $\operatorname{ar} 949 \mathrm{pt} 03$ & Migmatitic kinzigites & 108 & 693 & 757 & 853 \\
\hline $\operatorname{ar} 949 \mathrm{pt} 03$ & Migmatitic kinzigites & 130 & 703 & 780 & 878 \\
\hline $\operatorname{ar} 949 \mathrm{pt} 03$ & Migmatitic kinzigites & 134 & 706 & 784 & 883 \\
\hline $\operatorname{ar} 949 \mathrm{pt} 03$ & Migmatitic kinzigites & 118 & 693 & 767 & 865 \\
\hline $\operatorname{ar} 949 p t 04$ & Migmatitic kinzigites & 137 & 709 & 786 & 886 \\
\hline $\operatorname{ar} 949 \mathrm{pt} 04$ & Migmatitic kinzigites & 151 & 720 & 799 & 900 \\
\hline $\operatorname{ar} 949 \mathrm{pt} 04$ & Migmatitic kinzigites & 156 & 723 & 804 & 905 \\
\hline $\operatorname{ar} 949 \mathrm{pt} 04$ & Migmatitic kinzigites & 151 & 720 & 799 & 900 \\
\hline $\operatorname{ar} 949 p t 04$ & Migmatitic kinzigites & 152 & 721 & 800 & 901 \\
\hline
\end{tabular}


Table 1. Continued.

\begin{tabular}{|c|c|c|c|c|c|}
\hline \multirow{2}{*}{$\begin{array}{l}\text { Sample-crystal } \\
\text { number }\end{array}$} & \multirow[t]{2}{*}{ Lithology } & \multirow{2}{*}{$\begin{array}{c}\mathrm{Ti} \\
(\mathrm{ppm})\end{array}$} & \multicolumn{3}{|c|}{$T\left({ }^{\circ} \mathrm{C}\right), P=650 \mathrm{Mpa}$} \\
\hline & & & Thomas et al. (2010) & Wark and Watson (2006) & Huang and Audétat (2012) \\
\hline $\operatorname{ar} 949 p t 04$ & Migmatitic kinzigites & 146 & 716 & 795 & 895 \\
\hline $\operatorname{ar} 949 p t 04$ & Migmatitic kinzigites & 145 & 715 & 794 & 894 \\
\hline $\operatorname{ar} 949 p t 04$ & Migmatitic kinzigites & 144 & 714 & 793 & 893 \\
\hline $\operatorname{ar} 949 p t 04$ & Migmatitic kinzigites & 159 & 726 & 806 & 907 \\
\hline $\operatorname{ar} 949 p t 04$ & Migmatitic kinzigites & 151 & 720 & 799 & 900 \\
\hline ar949pt04 & Migmatitic kinzigites & 144 & 714 & 793 & 893 \\
\hline $\operatorname{ar} 949 p t 04$ & Migmatitic kinzigites & 158 & 725 & 805 & 907 \\
\hline $\operatorname{ar} 949 p t 04$ & Migmatitic kinzigites & 160 & 726 & 807 & 908 \\
\hline $\operatorname{ar} 949 p t 04$ & Migmatitic kinzigites & 152 & 721 & 800 & 901 \\
\hline $\operatorname{ar} 949 p t 04$ & Migmatitic kinzigites & 158 & 725 & 805 & 907 \\
\hline $\operatorname{ar} 949 p t 04$ & Migmatitic kinzigites & 155 & 723 & 803 & 904 \\
\hline $\operatorname{ar} 949 p t 04$ & Migmatitic kinzigites & 153 & 721 & 801 & 902 \\
\hline ar949pt04 & Migmatitic kinzigites & 158 & 725 & 805 & 907 \\
\hline $\operatorname{ar} 949 p t 04$ & Migmatitic kinzigites & 155 & 723 & 803 & 904 \\
\hline $\operatorname{ar} 949 \mathrm{pt} 04$ & Migmatitic kinzigites & 130 & 703 & 780 & 878 \\
\hline ar949 (average) & Migmatitic kinzigites & 147 & $717 \pm 10$ & $794 \pm 17$ & $884 \pm 20$ \\
\hline
\end{tabular}
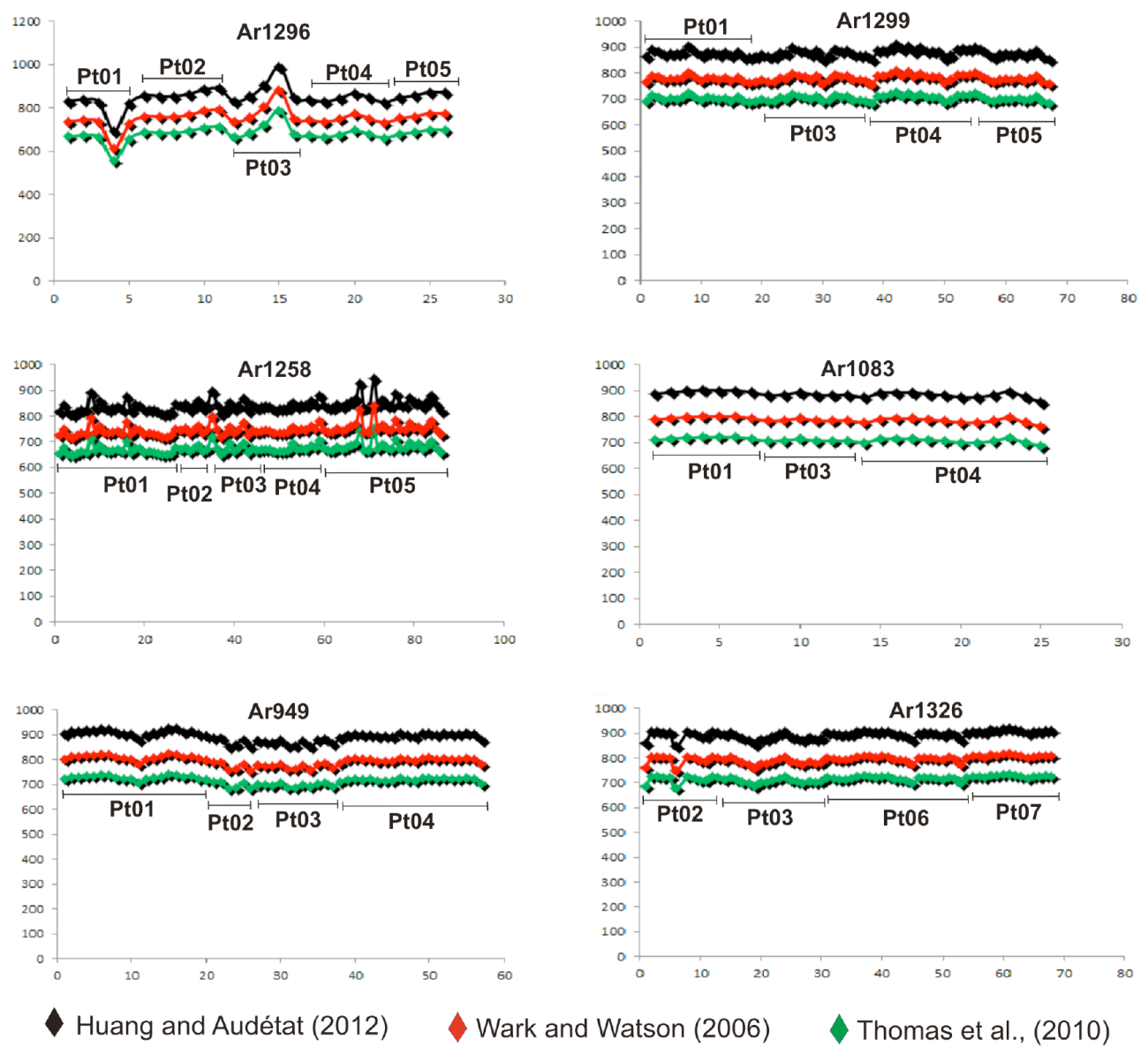

Figure 8. Calculated temperature ( $y$ axis) for each individual TitaniQ analysis ( $x$ axis $=$ analysis number in Table 1$)$ from the calibrations of Huang and Audétat (2012), Thomas et al. (2010) and Wark and Watson (2006). 
Table 2. Summarized geothermobarometric results for the studied rocks.

\begin{tabular}{lllllll}
\hline Identification & Analysis & Thermometer & $T\left({ }^{\circ} \mathrm{C}\right)$ & Reference & Barometer & $P(\mathrm{Mpa})$ \\
\hline \multirow{2}{*}{ Ar949 } & Core & grt-bt & $802-832$ & Ferry and Spear (1978) & GPBQ & \\
& Rim & grt-bt & $607-742$ & Ferry and Spear (1978) & GPBQ & $349-447$ \\
& Core & grt-crd & $797-824$ & Bhattacharya et al. (1988) & GPBQ & \\
& Rim & grt-crd & $756-789$ & Bhattacharya et al. (1988) & GPBQ & \\
\hline \multirow{2}{*}{ Ar1083 } & Core & grt-bt & $826-842$ & Ferry and Spear (1978) & GPBQ & $296-338$ \\
& Rim & grt-bt & $633-679$ & Ferry and Spear (1978) & GPBQ & $321-335$ \\
\hline \multirow{2}{*}{ Ar1076c } & Core & grt-bt & $765-835$ & Ferry and Spear (1978) & GPBQ & 704 \\
\hline \multirow{2}{*}{ Ar1076d } & Core & grt-bt & $721-814$ & Ferry and Spear (1978) & GPBQ & \\
& Rim & grt-bt & $558-668$ & Ferry and Spear (1978) & GPBQ & 559-612 \\
& Core & grt-opx & $792-831$ & Bhattacharya et al. (1991) & GPBQ & $651-652$ \\
\hline
\end{tabular}

by $\mathrm{Ti}$ in quartz. This suggests that post-crystallization cooling had little effect on the Ti content in quartz.

The temperatures obtained from Huang and Audétat's (2012) calibration are $50-100^{\circ} \mathrm{C}$ higher than those from the Thomas et al. (2010) and Wark and Watson calibrations (2006; Table 1). Huang and Audétat (2012) attributed the discrepancy between their calibration and Thomas et al. (2010) to the growth rate of quartz grains. Their experiments were carried out on quartz grains whose growth rates were 4$10 \mu \mathrm{mday}^{-1}$, and they concluded that the quartz grains of the Thomas et al. (2010) experiments, with $440 \mu \mathrm{m}$ to $\sim 1 \mathrm{~mm}$ in size, would have a growth rate $10-50$ times faster $(\sim 50-100$ to $250-500 \mu \mathrm{m} \mathrm{day}^{-1}$ ). According to the Huang and Audétat (2012) experiments, higher growth rates artificially increase the incorporation of titanium into quartz, and this tends to shift the resulting calibration toward lower temperatures.

We can expect grain growth in anatexites to be rather slow, especially when the cooling rate is low, and thus that temperatures computed with Huang and Audétat (2012) will be more reliable. However, considering the uncertainty that remains in the calibration of the TitaniQ geothermobarometers, in our estimate of the viscosity of the anatexites, we will use a conservative value between those obtained from the Thomas et al. (2010), Wark and Watson (2006) and Huang and Audétat (2012) calibrations. We roughly evaluate the temperature at which quartz grains started to crystalize using TitaniQ temperatures calculated from Wark and Watson (2006).

Temperatures obtained from Wark and Watson's (2006) calibration range from 700 to $810^{\circ} \mathrm{C}$, with individual average values between 750 and $800^{\circ} \mathrm{C}$ (Table 1; Fig. 8). For each sample, the analyzed grains define a temperature range that likely represents the minimum temperature interval over which quartz crystallized from the magma during cooling and solidification of the anatectic domain. Data lying significantly outside this domain of temperatures and contrasting strongly with the temperature of neighboring points of analysis may be regarded as out of equilibrium, and thus have not been taken into account further. The upper limit of this temperature interval is considered to represent the initial stage of interstitial quartz crystallization, and consequently the minimum value for the peak temperature to which the anatectic domain was submitted. Samples Ar949, Ar1083, Ar1299 and Ar1326 strongly suggest a minimum temperature of $\sim 800^{\circ} \mathrm{C}$ or slightly above. Samples Ar1258 and Ar1296 suggest a slightly lower temperature in the range $750-800^{\circ} \mathrm{C}$.

Migmatites and silicate melts containing Al-rich minerals are usually generated by partial melting of metasedimentary rocks in the crust at minimum temperatures $\geq 700^{\circ} \mathrm{C}$ (e.g., MacRae and Nesbit, 1980; White et al., 2003). The viscosity of resulting melts has been estimated at $\sim 10^{4.5} \mathrm{Pas}$ (Scaillet et al., 1998) or at $10^{3}$ to $10^{6} \mathrm{Pas}$ (Clemens and Petford, 1999). The viscosity of solid rocks differs from the viscosity of their melts by several orders of magnitude. Therefore, the viscosity of partially molten rocks varies widely, depending on the melt fraction, the viscosities of the molten and crystalline fractions, and on the shape and spatial distribution of solid particles (e.g., Arzi, 1978). Experiments suggest that the effect of the melt fraction on the viscosity of migmatite dominates the effects of temperature, pressure and stress, which vary less abruptly (Arzi, 1978; Rosenberg and Handy, 2005). According to Arzi (1978), a rock containing a melt fraction of $20 \pm 10 \%$ would reach the critical melt percentage (RCMP), above which, due to the connectivity of the melt fraction, its viscosity would decrease and rapidly approximate the melt's viscosity. In their compilation of experiments, Rosenberg and Handy (2005) suggest that the largest weakening resulting from partial melting occurs for melt fractions as low as $7 \%$.

The amount of titanium preserved in quartz is in equilibrium with the temperature prevailing when quartz crystallized from the melt. The peak temperature reached by the anatexites was therefore higher than the temperatures 
Table 3. Summarized oxide mineral chemistry for the studied rocks, including the major element composition (wt $\%$ ) of selected garnet, biotite, plagioclase, orthopyroxene and cordierite. ${ }^{*}-=$ content lower than the detection limit.

\begin{tabular}{|c|c|c|c|c|c|c|c|c|c|c|c|c|c|}
\hline & Phase & $\mathrm{SiO}_{2}$ & $\mathrm{Al}_{2} \mathrm{O}_{3}$ & $\mathrm{TiO}_{2}$ & $\mathrm{Na}_{2} \mathrm{O}$ & $\mathrm{MgO}$ & $\mathrm{FeO}$ & $\mathrm{K}_{2} \mathrm{O}$ & $\mathrm{CaO}$ & $\mathrm{Cr}_{2} \mathrm{O}_{3}$ & $\mathrm{MnO}$ & $\mathrm{NiO}$ & Total \\
\hline \multirow{23}{*}{ 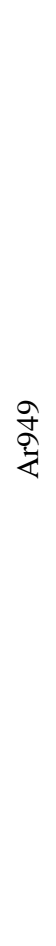 } & Garnet & 38.97 & 21.81 & 0.01 & - & 6.98 & 31.96 & 0.01 & 1.16 & 0.01 & 0.83 & - & 101.75 \\
\hline & & 38.69 & 21.82 & 0.04 & 0.01 & 7.35 & 31.52 & - & 1.17 & - & 0.75 & - & 101.35 \\
\hline & & 38.63 & 21.94 & 0.01 & 0.01 & 7.69 & 30.99 & - & 1.22 & 0.01 & 0.77 & 0.01 & 101.28 \\
\hline & & 38.70 & 21.91 & 0.02 & 0.01 & 7.85 & 31.03 & - & 1.24 & 0.02 & 0.80 & - & 101.58 \\
\hline & & 38.78 & 22.04 & 0.03 & - & 7.84 & 31.26 & - & 1.17 & 0.01 & 0.73 & 0.01 & 101.88 \\
\hline & & 38.54 & 21.92 & - & 0.02 & 7.68 & 31.06 & - & 1.15 & 0.05 & 0.78 & - & 101.20 \\
\hline & & 38.47 & 21.93 & 0.04 & 0.01 & 8.18 & 30.30 & - & 1.15 & 0.03 & 0.74 & - & 100.86 \\
\hline & & 38.62 & 21.97 & 0.04 & - & 8.13 & 30.62 & - & 1.19 & 0.03 & 0.74 & 0.01 & 101.36 \\
\hline & Biotite & 36.36 & 16.39 & 5.79 & 0.12 & 11.58 & 15.61 & 9.94 & 0.00 & 0.07 & 0.01 & 0.03 & 95.91 \\
\hline & & 36.43 & 16.16 & 6.08 & 0.15 & 13.67 & 12.59 & 9.97 & 0.01 & 0.09 & 0.00 & 0.04 & 95.19 \\
\hline & & 36.93 & 17.43 & 6.13 & 0.37 & 13.33 & 12.39 & 9.43 & 0.03 & 0.10 & 0.03 & 0.03 & 96.18 \\
\hline & & 37.09 & 17.18 & 5.11 & 0.21 & 13.91 & 12.47 & 9.88 & - & 0.12 & - & 0.02 & 96.00 \\
\hline & & 37.21 & 16.92 & 5.85 & 0.19 & 14.50 & 11.02 & 10.02 & 0.02 & 0.09 & - & 0.03 & 95.85 \\
\hline & & 36.45 & 17.32 & 5.29 & 0.34 & 12.85 & 12.40 & 9.32 & - & 0.06 & 0.03 & 0.04 & 94.10 \\
\hline & & 36.33 & 17.16 & 6.26 & 0.34 & 12.03 & 13.92 & 9.59 & - & 0.08 & 0.02 & 0.04 & 95.79 \\
\hline & Cordierite & 49.33 & 32.99 & 0.01 & 0.11 & 9.32 & 6.49 & 0.01 & 0.01 & 0.01 & 0.05 & - & 98.32 \\
\hline & & 49.40 & 33.39 & - & 0.08 & 9.73 & 6.42 & 0.00 & 0.01 & - & 0.07 & 0.03 & 99.12 \\
\hline & & 49.05 & 33.03 & - & 0.07 & 9.27 & 6.78 & 0.00 & - & 0.01 & 0.07 & 0.02 & 98.32 \\
\hline & & 49.29 & 33.10 & 0.02 & 0.07 & 9.30 & 6.66 & 0.00 & 0.02 & 0.01 & 0.07 & 0.02 & 98.55 \\
\hline & & 49.06 & 33.12 & - & 0.10 & 9.24 & 6.83 & 0.01 & 0.01 & - & 0.08 & 0.01 & 98.45 \\
\hline & Plagioclase & 59.77 & 24.92 & 0.01 & 7.78 & 0.01 & 0.02 & 0.26 & 6.74 & 0.01 & - & - & 99.52 \\
\hline & & 60.02 & 25.11 & 0.01 & 7.67 & 0.01 & - & 0.27 & 6.89 & 0.02 & - & - & 100.00 \\
\hline & & 60.15 & 24.93 & 0.03 & 7.71 & - & 0.01 & 0.29 & 6.73 & - & 0.01 & - & 99.86 \\
\hline \multirow{15}{*}{ 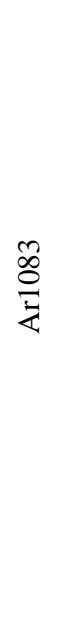 } & Garnet & 38.60 & 21.81 & 0.03 & - & 7.14 & 32.14 & - & 0.93 & 0.02 & 1.08 & - & 101.76 \\
\hline & & 38.73 & 22.01 & 0.02 & - & 7.07 & 31.51 & 0.01 & 0.93 & 0.03 & 1.08 & 0.02 & 101.40 \\
\hline & & 38.74 & 21.79 & 0.01 & - & 7.21 & 31.66 & - & 0.93 & 0.04 & 1.08 & 0.02 & 101.48 \\
\hline & & 39.32 & 22.14 & 0.02 & 0.01 & 7.06 & 31.69 & 0.01 & 0.95 & 0.03 & 0.03 & - & 102.28 \\
\hline & & 38.49 & 21.94 & - & - & 7.13 & 31.76 & - & 0.91 & 0.04 & 1.09 & - & 101.37 \\
\hline & Biotite & 35.55 & 17.27 & 6.27 & 0.36 & 13.44 & 12.17 & 9.57 & 0.00 & 0.08 & 0.03 & 0.04 & 94.79 \\
\hline & & 36.57 & 17.27 & 6.46 & 0.33 & 13.18 & 12.56 & 9.73 & - & 0.10 & 0.03 & 0.03 & 96.25 \\
\hline & & 36.92 & 17.41 & 6.35 & 0.31 & 12.77 & 12.74 & 9.80 & - & 0.07 & 0.03 & 0.01 & 96.41 \\
\hline & & 36.75 & 16.95 & 5.51 & 0.21 & 11.10 & 15.91 & 9.90 & 0.02 & 0.09 & 0.02 & 0.02 & 96.49 \\
\hline & & 36.91 & 16.70 & 5.51 & 0.11 & 11.32 & 15.48 & 10.02 & 0.01 & 0.08 & 0.03 & 0.01 & 96.17 \\
\hline & Plagioclase & 61.59 & 24.21 & 0.02 & 8.34 & 0.01 & - & 0.24 & 5.68 & - & - & - & 100.09 \\
\hline & & 62.29 & 23.76 & 0.04 & 8.51 & - & 0.02 & 0.31 & 5.23 & - & 0.03 & 0.01 & 100.19 \\
\hline & & 61.74 & 24.21 & 0.02 & 8.39 & - & 0.03 & 0.24 & 5.61 & 0.01 & - & - & 100.26 \\
\hline & & 61.24 & 24.30 & 0.02 & 8.48 & - & 0.09 & 0.16 & 5.87 & 0.02 & - & - & 100.19 \\
\hline & & 61.56 & 24.40 & 0.01 & 8.41 & - & 0.03 & 0.21 & 5.76 & 0.01 & 0.01 & - & 100.40 \\
\hline \multirow{7}{*}{\begin{tabular}{l}
0 \\
5 \\
0 \\
$\vdots$ \\
\hdashline
\end{tabular}} & Garnet & 38.33 & 21.71 & 0.04 & - & 5.80 & 32.45 & - & 2.11 & 0.04 & 1.30 & - & 101.78 \\
\hline & & 38.29 & 21.63 & 0.03 & - & 5.29 & 33.24 & - & 1.84 & - & 1.36 & - & 101.67 \\
\hline & Biotite & 36.18 & 16.23 & 4.82 & 0.14 & 10.51 & 18.24 & 9.78 & 0.00 & 0.06 & 0.05 & 0.03 & 96.04 \\
\hline & & 36.27 & 16.13 & 4.94 & 0.15 & 10.58 & 18.15 & 9.83 & 0.01 & 0.06 & 0.02 & 0.02 & 96.16 \\
\hline & & 36.27 & 16.35 & 4.78 & 0.15 & 11.42 & 17.23 & 9.75 & - & 0.06 & 0.02 & 0.05 & 96.09 \\
\hline & & 36.42 & 16.16 & 4.86 & 0.15 & 10.45 & 18.22 & 9.65 & - & 0.05 & 0.03 & 0.02 & 96.02 \\
\hline & Plagioclase & 59.97 & 25.40 & 0.02 & 7.60 & - & 0.08 & 0.19 & 7.10 & 0.03 & 0.01 & - & 100.40 \\
\hline
\end{tabular}


Table 3. Continued.

\begin{tabular}{|c|c|c|c|c|c|c|c|c|c|c|c|c|c|}
\hline & Phase & $\mathrm{SiO}_{2}$ & $\mathrm{Al}_{2} \mathrm{O}_{3}$ & $\mathrm{TiO}_{2}$ & $\mathrm{Na}_{2} \mathrm{O}$ & $\mathrm{MgO}$ & $\mathrm{FeO}$ & $\mathrm{K}_{2} \mathrm{O}$ & $\mathrm{CaO}$ & $\mathrm{Cr}_{2} \mathrm{O}_{3}$ & $\mathrm{MnO}$ & $\mathrm{NiO}$ & Total \\
\hline & \multirow[t]{14}{*}{ Garnet } & 37.86 & 21.43 & 0.05 & - & 5.71 & 32.38 & 0.01 & 2.09 & 0.03 & 1.29 & - & 100.85 \\
\hline & & 37.83 & 21.47 & 0.02 & 0.01 & 4.45 & 34.66 & 0.01 & 1.65 & 0.06 & 1.37 & - & 101.53 \\
\hline & & 37.93 & 21.63 & 0.03 & 0.01 & 6.02 & 31.58 & - & 2.17 & 0.03 & 1.28 & - & 100.68 \\
\hline & & 38.37 & 21.72 & 0.03 & - & 6.27 & 31.58 & 0.01 & 2.11 & 0.03 & 1.23 & - & 101.34 \\
\hline & & 37.53 & 21.34 & 0.02 & - & 5.06 & 33.53 & - & 1.80 & 0.02 & 1.32 & 0.02 & 100.63 \\
\hline & & 37.35 & 20.99 & 0.03 & 0.03 & 5.08 & 33.86 & - & 1.31 & 0.05 & 1.38 & - & 100.08 \\
\hline & & 37.55 & 21.26 & 0.01 & 0.01 & 5.27 & 33.13 & 0.01 & 1.70 & 0.03 & 1.36 & 0.01 & 100.33 \\
\hline & & 39.95 & 22.63 & - & 0.01 & 5.36 & 32.10 & - & 1.79 & 0.03 & 1.32 & - & 103.19 \\
\hline & & 38.41 & 21.64 & 0.04 & 0.02 & 6.15 & 31.28 & - & 1.94 & 0.04 & 1.20 & - & 100.72 \\
\hline & & 38.38 & 21.57 & 0.02 & 0.03 & 6.22 & 31.79 & - & 1.95 & 0.01 & 1.25 & 0.01 & 101.24 \\
\hline & & 38.27 & 21.64 & - & - & 6.29 & 31.89 & 0.01 & 1.90 & 0.04 & 1.26 & - & 101.29 \\
\hline & & 38.17 & 21.71 & 0.02 & 0.01 & 6.29 & 31.95 & - & 1.76 & 0.03 & 1.26 & - & 101.20 \\
\hline & & 38.28 & 21.43 & 0.01 & 0.02 & 6.15 & 31.34 & 0.02 & 1.76 & 0.05 & 1.26 & - & 100.32 \\
\hline & & 38.38 & 21.70 & - & - & 6.18 & 31.90 & - & 1.78 & 0.03 & 1.30 & - & 101.27 \\
\hline & \multirow[t]{13}{*}{ Biotite } & 36.05 & 16.21 & 4.71 & 0.12 & 11.09 & 17.59 & 9.82 & 0.01 & 0.08 & 0.03 & 0.01 & 95.72 \\
\hline \multirow{20}{*}{ 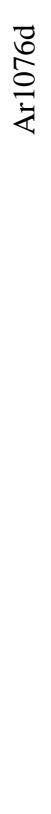 } & & 35.79 & 16.21 & 4.58 & 0.16 & 11.09 & 17.77 & 9.61 & 0.03 & 0.10 & 0.01 & 0.02 & 95.35 \\
\hline & & 36.25 & 16.21 & 4.59 & 0.13 & 11.65 & 17.26 & 9.87 & - & 0.05 & 0.03 & 0.04 & 96.09 \\
\hline & & 36.24 & 16.20 & 4.56 & 0.13 & 11.55 & 17.39 & 9.85 & 0.05 & 0.06 & 0.03 & 0.02 & 96.09 \\
\hline & & 36.30 & 16.20 & 4.55 & 0.19 & 13.38 & 14.35 & 9.70 & 0.01 & 0.08 & 0.02 & 0.02 & 94.80 \\
\hline & & 36.36 & 16.15 & 4.55 & 0.16 & 11.63 & 16.85 & 9.81 & - & 0.08 & 0.01 & 0.01 & 95.60 \\
\hline & & 36.20 & 16.08 & 4.54 & 0.14 & 11.40 & 17.71 & 9.83 & 0.02 & 0.05 & 0.02 & 0.04 & 96.01 \\
\hline & & 36.33 & 16.22 & 4.33 & 0.14 & 11.41 & 17.31 & 9.75 & 0.01 & 0.06 & 0.02 & 0.03 & 95.62 \\
\hline & & 36.42 & 16.22 & 4.32 & 0.10 & 11.33 & 17.01 & 9.79 & 0.01 & 0.06 & 0.03 & 0.03 & 95.33 \\
\hline & & 35.87 & 16.22 & 4.41 & 0.11 & 11.46 & 17.22 & 9.74 & 0.01 & 0.10 & 0.01 & 0.01 & 95.17 \\
\hline & & 36.28 & 16.27 & 4.42 & 0.13 & 11.31 & 17.27 & 9.82 & 0.01 & 0.07 & - & 0.03 & 95.58 \\
\hline & & 36.28 & 16.19 & 4.45 & 0.14 & 11.37 & 17.04 & 9.70 & 0.02 & 0.06 & 0.03 & 0.02 & 95.30 \\
\hline & & 35.87 & 16.09 & 4.31 & 0.13 & 11.65 & 17.54 & 9.75 & 0.02 & 0.10 & 0.03 & 0.02 & 95.51 \\
\hline & \multirow[t]{4}{*}{ Opx } & 49.30 & 2.95 & 0.06 & 0.01 & 15.59 & 30.97 & - & 0.15 & 0.02 & 0.49 & - & 99.53 \\
\hline & & 48.99 & 2.91 & 0.09 & - & 15.75 & 30.89 & 0.01 & 0.15 & - & 0.49 & - & 99.30 \\
\hline & & 49.75 & 2.76 & 0.07 & - & 15.94 & 30.60 & - & 0.15 & 0.01 & 0.45 & - & 99.72 \\
\hline & & 49.33 & 3.04 & 0.07 & - & 15.67 & 31.17 & - & 0.10 & 0.01 & 0.47 & 0.02 & 99.89 \\
\hline & \multirow[t]{4}{*}{ Plagioclase } & 59.39 & 24.99 & 0.04 & 7.55 & - & 0.06 & 0.27 & 6.71 & 0.04 & - & 0.01 & 99.07 \\
\hline & & 59.93 & 24.87 & 0.03 & 7.49 & - & 0.09 & 0.14 & 6.38 & - & 0.01 & - & 98.93 \\
\hline & & 59.41 & 24.91 & 0.01 & 7.70 & - & 0.03 & 0.31 & 6.70 & - & 0.01 & - & 99.06 \\
\hline & & 59.17 & 24.80 & 0.02 & 7.49 & - & 0.03 & 0.29 & 6.67 & - & - & 0.03 & 98.50 \\
\hline
\end{tabular}

calculated using the TitaniQ calibration $\left(750-800^{\circ} \mathrm{C}\right) . P-T$ estimates from exchange geothermobarometers suggest that the minimum temperatures and pressures for peak metamorphic conditions are recorded in samples of migmatitic kinzigites from which are derived, at least partly, the anatexites at $\sim 800 \pm 50^{\circ} \mathrm{C}$ and $\sim 650-700 \pm 100 \mathrm{MPa}$. They are also consistent with estimates from Munhá et al. $\left(2005 ; \sim 820^{\circ} \mathrm{C}\right.$, $650 \mathrm{MPa}$ ). A temperature of $800^{\circ} \mathrm{C}$ for the anatexites seems therefore to be a conservative value for discussing their rheological characteristics further.

We have estimated the solid-liquid proportion in the neosomes of the anatexites using the rhyolite MELTS software (Gualda et al., 2012) for temperature and pressure in the ranges $800-850{ }^{\circ} \mathrm{C}$ and $600-700 \mathrm{MPa}, \mathrm{H}_{2} \mathrm{O}$ content $1-2 \%$, and bulk rock composition of seven samples of neosomes
(Table 4). The proportion of liquid in the system, inferred using these parameters, is systematically larger than $28 \%$, and may reach up to $\sim 50 \%$. The viscosity of the liquid phase varies in the range $10^{5}-10^{6} \mathrm{Pas}$. MELTS predicts progressive crystallization of quartz (in a ratio inversely proportional to the amount of liquid in the system) with decreasing temperature. Such values are in agreement with the ubiquitous presence of films of quartz or feldspar separating inherited crystals, and also with the stromatic, schollen and locally nebulitic aspect of the anatexites (e.g., Sawyer, 2008). We may therefore consider that at conservative $P-T$ values of $800{ }^{\circ} \mathrm{C}$ and $650 \mathrm{MPa}, 30 \%$ liquid present in the neosome is a reasonable value that may be used to evaluate the viscosity of the magmatic suspension. For such a proportion of solids in the magma (70\%), MELTS does not compute the viscosity 
Table 4. Bulk rock composition of neosomes of representative anatectic rocks.

\begin{tabular}{lccccccc}
\hline $\mathrm{Wt} \%$ & Ar1113 & Ar952 & Ar1171 & Ar1172 & Ar1178 & Ar1184 & Ar1185 \\
\hline $\mathrm{SiO}_{2}$ & 72.53 & 72.43 & 71.61 & 72.81 & 71.45 & 69.73 & 71.18 \\
$\mathrm{Al}_{2} \mathrm{O}_{3}$ & 13.78 & 14.59 & 14.31 & 13.89 & 14.29 & 15.11 & 14.59 \\
$\mathrm{Fe}_{2} \mathrm{O}_{3}$ & 2.65 & 1.62 & 2.94 & 2.26 & 2.95 & 3.11 & 2.81 \\
$\mathrm{MgO}$ & 0.74 & 0.45 & 0.68 & 0.53 & 0.75 & 0.90 & 0.69 \\
$\mathrm{CaO}$ & 2.00 & 0.76 & 1.37 & 1.46 & 1.81 & 2.51 & 1.67 \\
$\mathrm{Na} 2 \mathrm{O}$ & 2.42 & 2.57 & 2.46 & 2.55 & 2.52 & 2.85 & 2.73 \\
$\mathrm{~K}_{2} \mathrm{O}$ & 4.37 & 5.92 & 5.50 & 5.30 & 4.83 & 4.11 & 4.90 \\
$\mathrm{TiO}_{2}$ & 0.40 & 0.33 & 0.38 & 0.32 & 0.43 & 0.52 & 0.38 \\
$\mathrm{P}_{2} \mathrm{O} 5$ & 0.19 & 0.28 & 0.12 & 0.19 & 0.21 & 0.21 & 0.24 \\
$\mathrm{MnO}$ & 0.05 & 0.01 & 0.04 & 0.03 & 0.05 & 0.05 & 0.04 \\
$\mathrm{LOI}$ & 0.8 & 0.9 & 0.5 & 0.6 & 0.6 & 0.8 & 0.7 \\
$\mathrm{Sum}$ & 99.89 & 99.86 & 99.92 & 99.93 & 99.89 & 99.86 & 99.90 \\
\hline
\end{tabular}

of the system. We therefore roughly estimated this viscosity using the Roscoe (1952) equation:

$\eta_{\mathrm{e}}=\eta(1-1.35 \varphi)^{-2.5}$,

where $\eta_{\mathrm{e}}$ is the effective viscosity of a suspension made of a liquid of viscosity $\eta$, and $\varphi$ is the solid fraction of spherical particles. The computed viscosity of the suspension (neosome) varies between $\sim 10^{8}$ and $\sim 10^{10} \mathrm{Pas}$ for $30 \%$ of the liquid phase, with viscosities of $10^{5}$ and $10^{6} \mathrm{Pas}$. If a rather large diversity of grain sizes is taken into account, the viscosity of the suspension decreases by about 1 order of magnitude (e.g., Kansal et al., 2002).

Restitic bodies in the anatectic domain of the Araçuaí belt, independent of their size ( $\mathrm{cm}$ to $\mathrm{km}$ ), are systematically immersed in neosome and anatectic granites that form a network of magma (Fig. 4) connected at the regional scale. This supports the finding that the viscosity of the partially molten crust was closer to the magma viscosity than to the solid-rock viscosity (e.g., Sawyer, 2008) and, in any case, significantly lower than the viscosity of $10^{17} \mathrm{Pas}$ (Grujic et al., 1996) or $10^{19} \mathrm{~Pa}$ (Beaumont et al., 2004), below which estimates from the Himalayan orogen predict an increasing probability of large-scale horizontal flow being triggered in response to gravity forces.

The anatectic crust of the Araçuaí belt was formed during the east-west (present-day orientation) collision between the South American and African plates that lasted more than 50 Myr (Schmitt et al., 2004; Vauchez et al., 2007; Pedrosa-Soares et al., 2008; Petitgirard et al., 2009; Monié et al., 2012). Seismic receiver functions suggest that the current crustal thickness of the Araçuaí-Ribeira belt is $\sim 32-41 \mathrm{~km}$ (Assumpção et al., 2013). Considering the pressure estimates obtained in this study and by Munhá et al. (2005) for the minimum conditions for metamorphic peaks of the migmatitic kinzigites (650-700 MPa), we can infer that approximately $25 \mathrm{~km}$ of the continental crust thickness have been removed. It follows that the crust thickness reached at least $60 \mathrm{~km}$ during the continent-continent collision, suggesting high to- pography. Such a thick crust, an abnormally high geotherm $\left(\geq 30^{\circ} \mathrm{C} \mathrm{km}^{-1}\right)$ and a slow cooling rate $\left(\leq 5^{\circ} \mathrm{C} \mathrm{Myr}^{-1}\right.$; Petitgirard et al., 2009) resulted in widespread partial melting of the middle to lower crust and a decrease in the thermal conductivity of the middle crust, enhancing abnormally high temperatures. These conditions dropped the viscosity of the neosome to $\sim 10^{10} \mathrm{Pas}$, supporting the finding that the viscosity of the anatectic middle crust was low enough $\left(\ll 10^{17} \mathrm{Pas}\right)$ to enable a gravity-induced component of flow. Considering the cooling rate, the large volume of melt produced during orogeny, and the latent heat of crystallization release by this anatectic layer, we can hypothesize that this low-viscosity layer remained so over a very long period (tens of Myr), its viscosity increasing slowly with the decrease in temperature. This supports the proposal that the gravitydriven deformation may have combined with deformation due to east-west convergence between the South American and African proto-continents, resulting in a complex 3-D flow of the anatectic middle crust.

\section{Conclusions}

Temperature estimates from the middle crust of the Araçuaí belt acquired using the titanium-in-quartz geothermometer in anatectic rocks are similar to those obtained from exchange geothermometry in neighboring kinzigites that have mineral assemblages suitable for such analysis. This good agreement supports that the TitaniQ geothermometer provides reliable crystallization temperatures of quartz in anatectic rocks. These estimates point to minimum temperature and pressure conditions for metamorphic peaks above $800^{\circ} \mathrm{C}$ and $650-700 \mathrm{MPa}$. Solid/liquid fractions computed using conservative $P$ and $T$ values and the chemical compositions of several anatexites suggest that at least $30 \%$ of the melt was present when the middle crust reached $800^{\circ} \mathrm{C}$. The proportion of melt under peak metamorphism conditions during orogeny was certainly higher than $30 \%$, a proportion consistent with the presence of in situ leucosomes and 
stromatic, nebulitic and schlieren structures in the anatexites. Such a proportion of melt is large enough to promote melt interconnectivity, as mentioned by Arzi (1978) and Rosenberg and Handy (2005), for instance. Rough estimates suggest that the partially molten middle crust had a viscosity significantly lower than the $10^{17}-10^{19} \mathrm{Pas}$ that is suggested by Grujic et al. (1996) and Beaumont et al. (2004) as the upper limit below which a rheologically weak crust is prone to being deformed through gravitational flow during orogeny. Therefore, considering the size $(\sim 300 \mathrm{~km} \times 50-100 \mathrm{~km})$ of the anatectic domain of the Araçuaí belt, the large orogenic thickness of the crust, the abnormally high temperature gradient, the low cooling rate, and the transient low viscosity of the partially molten middle crust, the complex flow pattern that characterizes the anatectic domain of the Araçuaí belt (e.g., Cavalcante et al., 2013) may result from the deformation of the low-viscosity middle crustal anatectic layer through gravitydriven escape flow associated with convergence-driven shortening.

Acknowledgements. GCGC is grateful to the Fundação de Amparo à Pesquisa do Estado de São Paulo (FAPESP, Brazil) project 2010/03537-7 and to the Coordenação de Aperfeiçoamento Pessoal de Nível Superior (CAPES, Brazil) project 419011-4 for the granting of $\mathrm{PhD}$ scholarships. We are indebted to Jean-Luc Bouchez, Loïc Labrousse and Djordje Grujic and one anonymous reviewer for their criticisms that helped substantially to improve this manuscript. Additional assistance by Bénédicte Cenki-Tok, Lucelene Martins, Fleurice Parat and Carlos Eduardo is greatly appreciated. Doriane Delmas and Christophe Nevado are acknowledged for the preparation of high-quality polished thin sections.

Edited by: F. Rossetti

\section{References}

Alsdorf, D., Makovsky, Y., Zhao, W., Brown, L. D., Nelson, K. D., Klemperer, S., Hauck, M., Ross, A., Cogan, M., Clark, M., Che, J., and Kuo, J.: INDEPTH (International Deep Profiling of Tibet and the Himalaya) multichannel seismic reflection data; description and availability, J. Geophys. Res., 103, 26993-26999, 1998.

Arzi, A.: Critical phenomena in the rheology of partially melted rocks, Tectonophysics, 44, 173-184, 1978.

Assumpção, M., Bianchi, M., Julià, J., Dias, F. L., França, G. S., Nascimento, R., Drouet, S., Pavão, C. G., and Albuquerque, D. F.: Crustal thickeness map of Brazil: data compilation and main feautures, J. S. Am. Earth Sci., 43, 74-85, 2013.

Bastin, G. F., Loo, F. J. J., Vosters, P. J. C., and Vrolijk, J. W. G. A.: An iterative procedure for the correction of secondary fluorescence effects in eléctron probe microanalysis near phase boundaries, Spectrochim. Acta, 39, 1517-1522, 1984.

Beaumont, C., Jamieson, R. A., Nguyen, M. H., and Medvedev, S.: Crustal channel flows: 1 . Numerical models with applications to the tectonics of the Himalayan-Tibetan orogen, J. Geophys. Res., 109, 1-29, 2004.

Bhattacharya, A., Mazumdar, A. C., and Sen, S. K.: Fe-Mg mixing in cordierite: constraints from natural data and implications for cordierite-garnet geothermometry in granulites, Am. Mineral., 73, 338-344, 1988.

Bhattacharya, A., Krishnakumar, K. R., Raith, M., and Sen, S. K.: An improved set of $\mathrm{a}-\mathrm{X}$ parameters for $\mathrm{Fe}-\mathrm{Mg}-\mathrm{Ca}$ garnets and refinements of the orthopyroxene-garnet thermometer and the orthopyroxene-garnet-plagioclase-quartz barometer, J. Petrol., 32, 629-656, 1991.

Bodinier, J.-L., Merlet, C., Bedini, R. M., Simien, F., Remaidi, M., and Garrido, C. J.: Distribution of $\mathrm{Nb}$, Ta and other highly incompatible trace elements in the lithospheric mantle: the spinel paradox, Geochim. Cosmochim. Ac., 60, 545-550, 1996.

Cavalcante, G. C. G., Egydio-Silva, M., Vauchez, A., Camps, P., and Oliveira, E.: Strain distribution across a partially molten middle crust: insights from the AMS mapping of the Carlos Chagas Anatexite, Araçuaí belt (East Brazil), J. Struct. Geol., 55, 79-100, 2013.

Chen, L., Booker, J. R., Jones, A. G., Wu, N., Unsworth, M. J., Wei, W., and Tan, H.: Electrically conductive crust in southern Tibet from INDEPTH magnetotelluric surveying, Science, 274, 1694 1696, 1996.

Clemens, J. D. and Petford, N.: Granitic melt viscosity and silicic magma dynamics in contrasting tectonic settings, J. Geol. Soc. Lond., 156, 1057-1060, 1999.

Cunningham, W. D., Marshak, S., and Alkmin, F. F.: Structural style of basin inversion at mid-crustal levels: two transects in the internal zone of the Brasiliano Araçuaí belt, Minas Gerais, Brazil, Precambrian Res., 77, 1-15, 1996.

Egydio-Silva, M., Vauchez, A., Bascou, J., and Hippertt, J.: Hightemperature deformation in the Neoproterozoic transpressional Ribeira belt, southeast Brazil, Tectonophysics, 352, 203-224, 2002.

Ehrlich, K., Vers, E., Kirs, J., and Soesoo, A.: Using a titaniumin-quartz geothermometer for crystallization temperature estimation of the Palaeoproterozoic Suursaari quartz porphyry, Est. J. Earth Sci., 61, 195-204, 2012.

Ferry, J. M. and Spear, F. S.: Experimental calibration of the partitioning of $\mathrm{Fe}$ and $\mathrm{Mg}$ between biotite and garnet, Contrib. Mineral. Petr., 66, 113-117, 1978.

Fournier, C., Merlet, C., Dugne, O., and Fialin, M.: Standardless semi-quantitative analysis with WDS-EPMA, J. Anal. Atom. Spectrom., 14, 381-386, 1999.

Grujic, D., Casey, M., Davidson, C., Hollister, L. S., Kundig, R., Pavlis, T., and Schmid, S.: Ductile extrusion of the Higher Himalayan Crystalline in Bhutan: evidence from quartz microfabrics, Tectonophysics, 260, 21-43, 1996.

Grujic, D., Stipp, M., and Wooden, J. L.: Thermometry of quartz mylonites: importance of dynamic recrystallization on Ti-inquartz reequilibration, Geochem. Geophy. Geosy., 12, 1-19, 2011.

Gualda, G. A. R., Ghiorso, M. S., Lemons, R. V., and Carley, T. L.: Rhyolite-MELTS: a modified calibration of MELTS optimized for silica-rich, fluid-bearing magmatic systems, J. Petrol., 25, 116, doi:10.1093/petrology/egr080, 2012.

Huang, R. and Audétat, A.: The titanium-in-quartz (TitaniQ) thermobarometer: A critical examination and re-calibration, Geochim. Cosmochim. Ac., 84, 75-89, 2012.

Jamieson, R. A., Unsworth, M. J., Harris, N. B. W., Rosenberg, C. L., and Schulmann, K.: Crustal melting and the flow of mountains, Elemts, 7, 253-260, 2011. 
Kalfoun, F., Ionov, D., and Merlet, C.: HFSE residence and Nb-Ta ratios in metasomatised, rutile-bearing mantle peridotites, Earth Planet. Sc. Lett., 199, 49-65, 2002a.

Kalfoun, F., Merlet, C., and Ionov, D.: Determination of Nb, Ta, $\mathrm{Zr}$ and $\mathrm{Hf}$ in micro-phases at low concentrations by EPMA, Mikrochim. Acta, 139, 83-91, 2002b.

Kansal, A. R., Torquato, S., and Stillinger, F. H.: Computer generation of dense polydisperse sphere packings, J. Chem. Phys., 117, 8212-8218, 2002.

Kohn, M. J. and Northrup, C. J.: Taking mylonite temperatures, Geology, 37, 47-50, doi:10.1130/G25081A.1, 2009.

Labrousse, L., Prouteau, G., and Ganzhorn, A.-C.: Continental exhumation triggered by partial melting at ultrahigh pressure, $\mathrm{Ge}-$ ology, 39, 1171-1174, 2011.

MacRae, N. D. and Nesbitt, H. W.: Partial melting of common metasedimantary rocks: an mass balance approach, Contrib. Mineral. Petr., 75, 21-26, 1980.

McNamara, D. E., Owens, T. J., and Walter, W. R.: Propagation characteristics of $\mathrm{Lg}$ across the Tibetan plateau, B. Seismol. Soc. Am., 86, 457-469, 1996.

Merlet, C.: An accurate computer correction program for quantitative electron probe microanalysis, Mikrochim. Acta, 114/115, 363-376, 1994.

Merlet, C. and Bodinier, J. L.: Electron microprobe determination of minor and trace transition elements in silicate mineral: a method and its application to mineral zoning in the peridotite nodule phn 1611, Chem. Geol., 83, 55-69, 1990.

Mondou, M., Egydio-Silva, M., Vauchez, A., Raposo, M. I. B., Bruguier, O., and Oliveira, A. F.: Complex, 3-D strain patterns in a synkinematic tonalite batholith from the Araçuaí Neoproterozoic orogen (Eastern Brazil): evidence from combined magnetic and isotopic chronology studies, J. Struct. Geol., 39, 158-179, doi:10.1016/j.jsg.2012.02.015, 2012.

Monié, P., Bosch, D., Bruguier, O., Vauchez, A., Rolland, Y., Nsungani, P., and Buta Neto, A.: The late Neoproterozoic/Early Palaezoic evolution of the West Congo Belt of NW Angola: Geochronological (U-Pb and $\mathrm{Ar}-\mathrm{Ar}$ ) and petrostructural constraints, Terra Nova, 24, 238-247, 2012.

Munhá, J. M. U., Cordani, U. G., Tassinari, C. C. G., and Palacios, T.: Petrologia e termocronologia de gnaisses migmatíticos da faixa de dobramentos araçuaí (espirito santo, brasil), Revista Brasileira de Geociências, 35, 123-134, 2005.

Negrini, M., Stunitz, H., Berger, A., and Morales, L. F. G.: The effect of deformation on the TitaniQ geothermobarometers: an experimental study, Contrib. Mineral. Petr., 167, 982, doi:10.1007/s00410-014-0982-x, 2014.

Nelson, K. D., Zhao, W., Brown, L. D., Kuo, J., Che, J., Liu, X., Klemperer, S. L., Makovsky, Y., Meissner, R., Mechie, J., Kind, R., Wenzel, F., Ni, J., Nabelek, J., Chen, L., Tan, H., Wei, W., Jones, A. G., Booker, J., Unsworth, M., Kidd, W. S. F., Hauck, M., Alsdorf, D., Ross, A., Cogan, M., Wu, C., Sandvol, E. A., and Edwards, M.: Partially molten middle crust beneath southern Tibet; synthesis of Project INDEPTH results, Science, 274, 1684-1688, 1996.

Oliveira, M.-J. R., Pinto, C. P., Féboli, W. L., and Alves dos Santos, R.: Projeto Leste - Relatorio mapa integrado 1 : 500000 - Geologia estrutural e tectônica, CPRM - COMIG, Belo Horizonte, 2000 .
Pedrosa-Soares, A. C., Queiroga, G. N., Gradim, C. T., Roncato, J. G., Novo, T. A., Jacobsohn, T., and Silva, K. L.: Programa Geologia do Brasil, Mapeamento geológico e nota explicativa da Folha Mantena (SE-24-Y-a-vi), escala $1: 100000$, Contrato CPRM 059/PR/05- UFMG (iGC/CPMC), CD-ROM, 2006.

Pedrosa-Soares, A. C., Alkmim, F. F., Tack, L., Noce, C. M., Babinski, M., Silva, L. C., and Martins-Neto, M. A.: Similarities and differences between the Brazilian and African counterparts of the Neoproterozoic Araçuaí-West Congo orogen, Geological Society, London, Special Publications, 294, 153-172, 2008.

Petitgirard, S., Vauchez, A., Egydio-Silva, M., Bruguier, O., Camps, P., Monié, P., Babinski, M., and Mondou, M.: Conflicting structural and geochronological data from the Ibituruna quartz-syenite (SE Brazil): Effect of protracted "hot" orogeny and slow cooling rate?, Tectonophysics, 477, 174-196, doi:10.1016/j.tecto.2009.02.039, 2009.

Roscoe, R.: The viscosity of suspensions of rigid spheres, Brit. J. Appl. Phys., 13, 267-269, 1952.

Rosenberg, C. L. and Handy, M. R.: Experimental deformation of partially melted granite revisited: implications for the continental crust, J. Metamorph. Geol., 23, 19-28, 2005.

Sawyer, E. W.: Working with migmatites, Mineralogical Association of Canada, Short course series, 38, 158 pp., 2008.

Scaillet, B., Holtz, F., Whittington, A., and Pichavant, M.: Phase equilibrium constraints on the viscosity of silicic magmas, 1. Volcanic-plutonic comparison, J. Geophys. Res., 103, 27257 27266, 1998.

Schmitt, R. S., Trouw, R. A. J., Van Schmus, W. R., and Pimentel, M. M.: Late amalgamation in the central part of West Gondwana: new geochronological data and the characterization 10 of a Cambrian collisional orogeny in the Ribeira Belt (SE Brazil), Precambrian Res., 133, 29-61, 2004.

Storm, L. C. and Spear, F. S.: Application of the titanium-in-quartz thermometer to pelitic migmatites from the Adirondack Highlands, New York, J. Metamorph. Geol., 27, 479-494, 2009.

Thomas, J. B., Watson, E. B., Spear, F. S., Shemella, P. T., Nayak, S. K., and Lanzirotti, A.: TitaniQ under pressure: the effect of pressure and temperature on the solubility of Ti in quartz, Contrib. Mineral. Petr., 160, 743-759, 2010.

Trompette, R.: Geology of Western Gondwana (2000-500 Ma), Balkema, Rotterdam, 350 pp., 1994.

Unsworth, M. J., Jones, A. G., Wei, W., Marquis, G., Gokarn, S. G., and Spratt, J. E. (INDEPTHMT TEAM): Crustal rheology of the Himalaya and Southern Tibet inferred from magnetotelluric data, Nature, 438, 78-81, 2005.

Vauchez, A., Egydio-Silva, M., Babinski, M., Tommasi, A., Uhlein, A., and Liu, D.: Deformation of a pervasively molten middle crust: insights from the Neoproterozoic Ribeira-Araçuaí orogen (SE Brazil), Terra Nova, 19, 278-286, 2007.

Wark, D. A. and Watson, E. B.: TitaniQ: a titanium-in-quartz geothermometer, Contrib. Mineral. Petr., 152, 743-754, 2006.

White, R. W., Powell, R., and Clarke, G. L.: Prograde Metamorphic Assemblage Evolution during Partial Melting of Metasedimentary Rocks at Low Pressures: Migmatites from Mt Stafford, Central Australia, J. Petrol., 44, 1937-1960, 2003.

Wu, C. M., Zhang, J. Z., and Ren, L. D.: Empirical Garnet-BiotitePlagioclase-Quartz (GBPQ) geobarometry in medium- to highgrade metapelites, J. Petrol., 45, 1907-1921, 2004. 